\title{
A search for spectroscopic binaries among Herbig Ae/Be stars $^{\star, \star \star}$
}

\author{
P. Corporon and A.-M. Lagrange \\ Laboratoire d'Astrophysique de l'Observatoire de Grenoble, BP. 53, F-38 041 Grenoble Cedex, France
}

Received March 4; accepted November 17, 1998

\begin{abstract}
We present the results of a spectroscopic survey of binaries among 42 bright $\left(m_{V}<11\right)$ Herbig $\mathrm{Ae} / \mathrm{Be}$ stars in both hemispheres. Radial velocity variations were found in 7 targets, 4 are new spectroscopic binaries. The Li I $6708 \AA$ absorption line (absent feature in simple HAeBe stars spectra) indicates the presence of a cooler companion in $6 \mathrm{HAeBe}$ spectrum binaries, 4 of which are new detections. Few stars classified as possible Herbig Ae/Be stars are not confirmed as such.

While for short-period $(P<100$ days) spectroscopic binaries, the observed binary frequency is $10 \%$, the true spectroscopic binary frequency for Herbig Ae/Be stars may be as high as $35 \%$.
\end{abstract}

Key words: binaries: spectroscopic - stars: early-type stars: pre-Main Sequence - stars: statistics

\section{Introduction}

\subsection{Multiplicity and evolution}

Multiplicity is a major issue in stellar astrophysics. Firstly, binary stars are very common among Main Sequence (MS) stars: half of the MS field stars are known to belong to multiple systems (see Garmany et al 1980 for O type stars; Abt et al. 1990 for B; Nordström et al. 1997 for F; Duquennoy \& Mayor 1991 for G; Mayor et al. 1992 for K; Leinert et al. 1997 for M). Thus, any stellar formation theory must explain this large abundance of multiple systems. Various mechanisms have been proposed to form

Send offprint requests to: P. Corporon

* Based on observations collected at the European Southern Observatory (ESO), La Silla, Chile and at the Observatoire de Haute-Provence (OHP), Saint-Michel l'Observatoire, France.

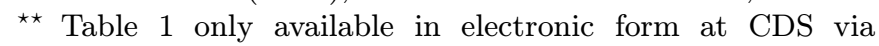
anonymous ftp to cdsarc.u-strasbg.fr (130.79.128.5) or via http://cdsweb.u-strasbg.fr/Abstract.html binaries (see a detailed review in Clarke 1996), but observations are needed to constrain further these models.

Secondly, a fundamental role of binary studies is to allow the direct determination of physical parameters. Noticeably, the stellar mass is only accessible through the observation of gravitationally bound pairs of stars, by straight application of gravitational law.

Main-Sequence (MS) binary stars are overall quite well studied. However, as orbits of binary systems evolve with time, it is mandatory to derive the properties of the systems during the pre-Main Sequence (PMS) phase. A major issue is to quantify the binary frequency $f b$ (the probability that a given object is multiple, Reipurth \& Zinnecker 1993) for young multiple objects, their separation distribution as well as their mass ratio.

Recent studies have shown that more than half of $\mathrm{T}$ Tauri stars, young stars having a mass $M<1.5 M_{\odot}$, are members of a binary or multiple system (Mathieu 1992; Leinert et al. 1993; Reipurth \& Zinnecker 1993; Prosser et al. 1994; Simon et al. 1995; Ghez et al. 1993, 1997; Brandner et al. 1996; Padgett et al. 1997). Whether there is an overabundance of low-mass PMS binaries versus MS binaries is still a matter of debate, due to the difficulties to compare both statistics obtained with different approaches. Not only the employed techniques are different, (MS stars were mainly spectroscopically searched for, while PMS binaries were searched with high angular resolution imaging), but also the wavelength domains are different (optical observations predominate for MS stars, while infrared (IR) surveys of the young objects, mostly embedded in dark regions, were used ipso facto). The only systematic effort aimed at finding new low-mass pre-Main Sequence binaries with visible spectroscopy dates back from Mathieu (1992).

The question is then whether the stellar density may have an effect on the formation of multiple systems. MS stars are thought to have been formed in OB associations or in dense clusters (Miller \& Scalo 1978; Lada et al. 1991), while PMS stars in T associations are formed in lower density environments which might enhance the 
production of binaries. However, Brandner et al. (1996) observed $\mathrm{T}$ Tauri stars in $\mathrm{OB}$ and $\mathrm{T}$ associations and concluded that the binary frequency is the same for MS and PMS low mass stars in the range of separation $120-1800 \mathrm{AU}$ (except for the Taurus-Auriga star forming region). Moreover, Padgett et al. (1997) using HST observations of PMS stars in dense clusters recently found a comparable binary frequency for dense clusters and lowdensity star-forming regions. They thus claim that multiplicity is not influenced by the local stellar density (at least in regions with densities ranging between 40 and 5000 stars $\left.\mathrm{pc}^{-3}\right)$.

Since the pioneering work of Mathieu (1992), selective mass determinations for some low-mass PMS binaries have been obtained (Padgett \& Stapelfeldt 1994; Welty 1995; Figueiredo 1997), but the sample of young multiple systems with orbital and physical parameters determined must be enlarged in order to test the early stages of stellar evolutionary models.

\subsection{Multiplicity of intermediate-mass PMS stars}

As pointed out by Hillebrand (1994), the intermediatemass PMS stars, namely Herbig Ae/Be (HAeBe) stars are found in various environments: in dense star forming regions (containing few tens of HAeBe plus a myriad of T Tauri stars), in lower density groups of young stellar objects ( 2 to $5 \mathrm{HAeBe}$ stars sharing the same birth place) and in isolated molecular cores (where a central HAeBe star and embedded young lower mass stars are found). Studying the binary frequency among HAeBe objects may help to better understand the relation between the direct environment and the multiplicity status of the stars during their earlier formation stages.

Besides reinforcing the binary frequency estimate, HAeBe binaries study is of great interest because direct mass determination are fervently required to test the stellar evolution models for young intermediate-mass stars.

To date, however, the binarity status of Ae/Be Herbig stars has been far less surveyed, probably because these stars form a class less homogeneous than $\mathrm{T}$ Tauri stars (Thé et al. 1994). Few recent studies using infrared imaging (Li et al. 1994; Leinert et al. 1997b; Pirzkal et al. 1997) have found a binary frequency (although based on limited samples) in excess by a factor 2 versus A/B type MS stars, (by considering G type MS stars degree of multiplicity $f b$, spectroscopically determined by Duquennoy and Mayor 1991 identical through the Main Sequence, as explained by Leinert et al. 1993).

Up to now, the pilot study made on the eclipsing and spectroscopic triple system TY CrA (Lagrange et al. 1993; Corporon et al. 1994, 1996; Beust et al. 1997, see also Casey et al. 1993, 1995, 1998) is the only work that led to the first direct determination of masses for a $\mathrm{HAeBe}$ multiple system.

\subsection{Aim of the present paper}

In this paper, we report the first results of a systematic high resolution spectroscopic search for HAeBe binaries. It is part of an extensive survey made on Herbig Ae/Be stars, the other facet being high angular resolution imaging of IR companions of HAeBe stars using Adaptative Optics systems (Bouvier et al. 1998; Corporon 1998). The two approaches are complementary: while the former gives access to the study of short orbital periods $(P=$ few hours to few months) of double stars, the latter covers the domain of longer periods ( $P=$ many years).

The present paper is structured as follows: in Sect. 2, we describe our sample, the instruments used as well as our observing strategy. We present in Sect. 3 the spectra of some known and new spectroscopic HAeBe binaries. Notes on individual sources are given in Sect. 4 and in Sect. 5 we discuss our results.

\section{Observations of the HAeBe binaries}

\subsection{The sample}

The observed Herbig Ae/Be stars were extracted from Table 1 of Thé et al. (1994) catalogue. We obtained high resolution spectra for 42 objects with $m_{V}<11$, consisting of our principal HAeBe sample. This sample represents about $70 \%$ of the HAeBe candidates with $m_{V}<11$ from Table 1 of Thé et al. (1994) catalogue. Most of the remaining stars were not observed either because they were already well studied or because they are strong photometric variables and out of our limit of our observing capabilities at their minimum brightness.

A sub-group of other $\mathrm{HAeBe}$ candidates listed in Tables 2 to 5 from the catalogue of Thé et al. (1994) were also studied (sample T2-T5). They were included in our survey because they could be observed in parallel to our main program. Those stars were observed not only to test the presence of a companion, but also to precise, when possible, their spectral type or to test their belonging to the Herbig Ae/Be stellar class.

We address in this paragraph the possible bias by the so-called Branch effect. Branch (1976) pointed out that a magnitude-limited sample favors the detection of doublelined spectroscopic binary (SB2). In other words, some binary stars could have been selected because their total magnitude is below the magnitude limit, whereas individual stars are fainter than the $m_{V}$ limit. Such systems should be removed when establishing the binary frequency of the sample. However, in our case with high mass primaries, unless the mass ratio is close to unity, the secondary component of a spectroscopic binary system contribute to few percents of the combined flux. We consider the example of the double-lined spectroscopic binary system TY CrA, adopting the most recent physical parameters determination by Casey et al. (1998): the primary 
(late-B type) has an effective temperature $T_{\mathrm{I}}$ of about $12000 \mathrm{~K}$ and a radius $R_{\mathrm{I}}=1.8 R_{\odot}$, while the secondary (late-G type) has $T_{\mathrm{II}} \approx 4900 \mathrm{~K}$ and $R_{\mathrm{II}}=2.1 R_{\odot}$. The flux ratio between the two stars is

$\frac{F_{\mathrm{II}}}{F_{\mathrm{I}}}=\frac{T_{\mathrm{II}}^{4} \times R_{\mathrm{II}}^{2}}{T_{\mathrm{I}}^{4} \times R_{\mathrm{I}}^{2}}=3.8 \%$.

Thus, in a HAeBe binary system with a primary of spectral type $\mathrm{A}$ or $\mathrm{B}$ and a lower-mass companion, the primary will be responsible for most of the observed flux. Therefore, the Branch effect is not significant in such cases. When both members of the spectroscopic HAeBe binary system have similar masses, and hence comparable luminosity, special attention should be paid to see whether or not one member of the system would have been in our sample or not.

Noticeably, we are faced with the large disparity in the location of the HAeBe type stars (some of them have a poorly determined distance). A distance limited criterion as considered by Duquennoy \& Mayor (1991) is hardly conceivable here to select a sufficiently large sample and to determine a reliable binary frequency for Herbig Ae/Be stars.

\subsection{Observing journal}

The spectroscopic survey, initiated in 1994, was carried out in the two hemispheres, using three different instruments which characteristics and data reduction procedures are described hereafter.

\subsubsection{Northern hemisphere}

Spectra of northern hemisphere HAeBe stars were taken with ÉLODIE and AURÉLIE, two spectrographs of the Observatoire de Haute-Provence (OHP), south of France.

AURÉLIE is a high resolution spectrograph mounted at the Coudé focus of the $1.52 \mathrm{~m}$ telescope. A detailed description is given in Gillet et al. (1994). The detector at the time of the observations was a Thomson double array and two different gratings were usually employed: \#7 with a resolution $R \approx 38000$ at $\lambda=6500 \AA$ and \#5 (2nd order) with $R \approx 70000$ at $\lambda=6500 \AA$. Typical exposure time was $1-1.5$ hour for our target stars, with a circular entrance hole of $3^{\prime \prime}$ on the sky. A continuous light-source provided the flat field exposures to correct the instrumental response. The flat-field images were chosen to have a level similar to that of the science exposures and were repeated each night. After subtraction of the bias and dark current (measured routinely during each night), the science exposure was divided by an average of ten suited flat-field images. Thorium and argon lamps were used for wavelength calibration, and numerous exposures were taken each night to monitor the stability of the spectrograph. The final wavelength calibration accuracy is $2 \mathrm{~km} \mathrm{~s}^{-1}$. The spectra were then transformed into the heliocentric rest frame and normalized to unity. Bad pixels or cosmic rays were removed by-hand. All the data reduction steps were performed with the ESO MIDAS software.

ÉLODIE, located at the $1.93 \mathrm{~m}$ OHP telescope, is a fiber-fed échelle spectrograph. The detector is a $1024 \times 1024$ Tektronics CCD. 67 orders are simultaneously recorded, giving in a single exposure a spectrum between 3906 and $6811 \AA$, at a resolution of 42000 . The optical layout as well as the reduction procedure can be found in Baranne et al. (1996). The fiber diameter is $2^{\prime \prime}$ on the sky and thorium and science spectra were obtained separately. Sky background was estimated in the inter-order space. Typical exposure times range from 0.5 to 1 hour. The final velocity calibration is better than $1 \mathrm{kms}^{-1}$. Noticeably, a program at the telescope automatically performs the data reduction: no further work but heliocentric velocity correction and normalization to unity is required to have a set of homogeneous spectra.

\subsubsection{Southern hemisphere}

Southern HAeBe stars of our sample were observed with the CES (Coudé Échelle spectrograph) fed by the $1.4 \mathrm{~m}$ CAT telescope (La Silla Observatory, Chile). Most of the observations were made under remote control from the ESO headquarters in Garching bei München. From December 1994 to October 1995, the short camera configuration and the CCD \#9 was used. Then we used the long camera and the new CCD \#38 allowing a larger $(\approx 60 \AA)$ spectral coverage in the Li I $6708 \AA$ region. The resolving power was set to 60000 . Typical integration time was $1-1.5$ hour, the rectangular slit dimensions were ranging from $5-10^{\prime \prime} \times 1-2^{\prime \prime}$. The data reduction procedure was identical to the one followed for the AURÉLIE data (except that the $2 \mathrm{D}$ - spectra were averaged perpendicularly to the dispersion).

The time distribution of the observations is a combination of high-frequency coverage (less than few days) and low-frequency coverage (greater than 1 year) over the final 3 years of the survey. Table 1 (only available in electronic form at the CDS) presents the observed stars.

Note: given the slit/fiber sizes and the extreme seeing values during the various observations $\left(0.7-2^{\prime \prime}\right)$, we estimate that a binary separated by less than $1.5^{\prime \prime}$ has been observed while integrating the flux from both components: such a system is thus considered "as a single target" in our spectroscopic observations. On the other hand, the primary HAeBe (brightest component in $V$ ) of a visual binary with separation greater than $1.5^{\prime \prime}$ was independently observed (i.e. without integrating the flux of its companion), when seeing conditions allowed it. 


\subsection{Spectral analysis}

We present here the two methods used to spectroscopically identify a HAeBe binary star.

\subsubsection{Search for Li I $6708 \AA$ absorption}

Martin (1994) quantitatively showed that the Li I $6707.8 \AA$ resonance doublet can be used to detect T Tauri companions of HAeBe stars. Indeed, in hot intermediate-mass stars, the Li I absorption line, extremely weak, is not detected, whereas in lower mass stars, Li I is detected (see Walter et al. 1988; Duncan \& Rebull 1996; Jones et al. 1996). If the spectroscopic signature of this element is present in the spectrum of a HAeBe star, it reveals then the presence of a young lower mass and cooler companion.

\subsubsection{Search for radial velocity variations}

In order to monitor the radial velocity $\left(V_{\text {rad }}\right)$ variations, we mainly used the He I 5876 and 6678, Na I 5890 and 5895, Si II 6347 and $6371 \AA$ lines. The center of the lines was measured by fitting simple Gaussian functions: the errors of such measurements are of the order of 5 to $10 \mathrm{~km} \mathrm{~s}^{-1}$, depending on the rotational velocity of the stars and its shape (if emission is also present and affects part of the photospheric line).

Note: although with ÉLODIE on-line crosscorrelation spectroscopy is possible, we did not used this option: our stars, hot objects with $7000<T_{\text {eff }}<30000 \mathrm{~K}$, display few lines in their visible spectrum, usually broadened by rapid rotation. Moreover, some lines may be filled-in by emission and show strong variations from night to night. Obviously, direct cross-correlation spectroscopy, as also proposed by Morse et al. (1991) for early-type stars, may not be appropriate for $\mathrm{Ae} / \mathrm{Be}$ stars.

\section{General results}

\subsection{HAeBe candidates detected as spectroscopic binaries}

Table 2 presents the 13 binary systems detected among our main sample, i.e. the HAeBe candidates from Table 1 of the catalogue of The et al. (1994).

A first group of 6 stars in Table 2 were identified as spectroscopic binaries thanks to the detection of the Li I $6708 \AA$ absorption line. This indicates the presence of a $\mathrm{T}$ Tauri companion. Their spectra are shown in Fig. 1.

Based on stellar models obtained with ATLAS9 (Kurucz 1993) and with solar metallicity, we computed synthetic spectra with SYNSPEC (Hubeny et al. 1994) for various spectral type (B0, B5, A0, A5, F0, F5, G5 and
Table 2. HAeBe candidates detected as spectroscopic binaries

\begin{tabular}{lccll}
\hline Star & $\begin{array}{c}\text { Li I line } \\
\text { detection }\end{array}$ & $\begin{array}{c}V_{\text {rad }} \\
\text { variations }\end{array}$ & $\begin{array}{l}\text { separation } \\
\rho_{\text {vis }}\end{array}$ & $\begin{array}{l}v \sin i \\
\left(\mathrm{~km} \mathrm{~s}^{-1}\right)\end{array}$ \\
\hline HK Ori & $\mathrm{Y}$ & $\mathrm{N}$ & $0.35^{\prime \prime \mathrm{a}, \mathrm{b}}$ & 150 \\
V380 Ori & $\mathrm{Y}$ & $\mathrm{N}$ & $0.15^{\prime \prime \mathrm{a}, \mathrm{b}}$ & 200 \\
V586 Ori & $\mathrm{Y}$ & $\mathrm{N}$ & $1.00^{\prime \prime} \mathrm{a}$ & $160^{\mathrm{e}}$ \\
NX Pup & $\mathrm{Y}$ & $\mathrm{N}$ & $0.13^{\prime \prime \mathrm{a}, \mathrm{d}}$ & $120^{\mathrm{e}}$ \\
HD 203024 & $\mathrm{Y}$ & $\mathrm{N}$ & $0.30^{\prime \prime \mathrm{a}}$ & 125 \\
MWC 863 & $\mathrm{Y}$ & $\mathrm{N}$ & $1.10^{\prime \prime} \mathrm{a}, \mathrm{c}$ & $100^{\mathrm{f}}$ \\
TY CrA & $\mathrm{Y}$ & $\mathrm{SB} 3$ & & $10^{\prime \prime \mathrm{b}}$ \\
T Ori & $\mathrm{N}$ & $\mathrm{Y}$ & $7.70^{\prime \prime}$ & 100 \\
HD 53367 & $\mathrm{N}$ & $\mathrm{SB} 1$ & $0.70^{\prime \prime} \mathrm{a}$ & $30^{\mathrm{f}}$ \\
MWC 300 & $\mathrm{N}$ & $\mathrm{Y}$ & & 50 \\
AS 442 & $\mathrm{N}$ & $\mathrm{SB} 1$ & & 70 \\
MWC 361 & $\mathrm{N}$ & $\mathrm{Y}$ & $2.50^{\prime \prime \mathrm{a}, \mathrm{c}}$ & $40^{\mathrm{e}}$ \\
MWC 1080 & $\mathrm{N}$ & $\mathrm{Y}$ & $0.80^{\prime \prime \mathrm{a}, \mathrm{b}, \mathrm{c}}$ & 200 \\
\hline
\end{tabular}

a Bouvier et al. 1998; b Leinert et al. 1997b; c Pirzkal et al. 1997; ${ }^{\mathrm{d}}$ Bernacca et al. 1993; ${ }^{\text {e }}$ Böhm \& Catala 1995; ${ }^{\mathrm{f}}$ Finkenzeller 1985.

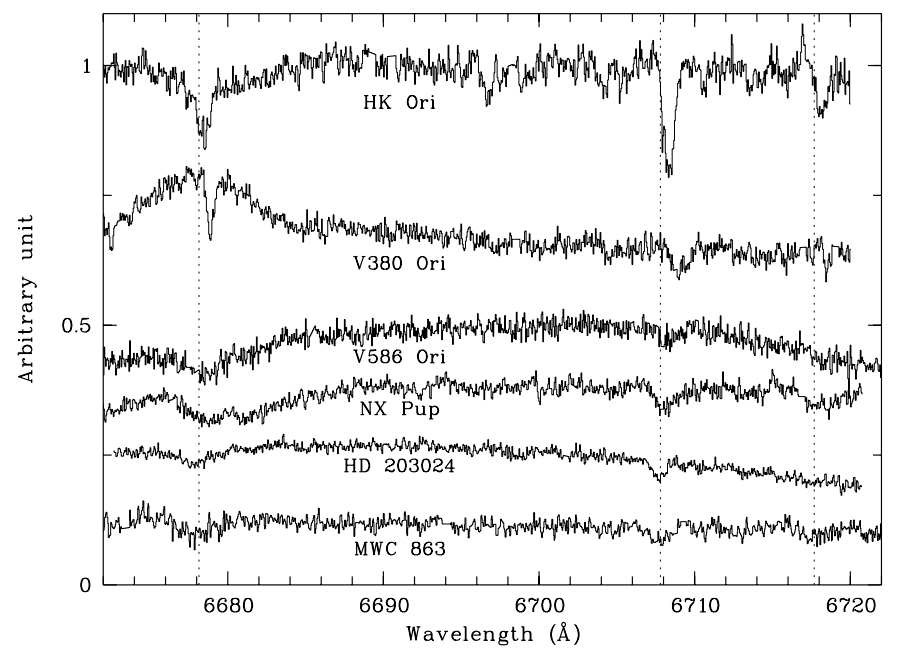

Fig. 1. HAeBe candidates identified as binaries thanks to the detection of Li I $6708 \AA$ line, attributed to a cooler companion. Laboratory positions of He I 6678.154, Li I 6707.800 and Ca I $6717.681 \AA$ lines are shown with doted lines

K5). The spectra are shown in Fig. 2 in the Li I $6708 \AA$ line region (the rotational velocity for each spectrum is $\left.V_{\text {rot }}=50 \mathrm{~km} \mathrm{~s}^{-1}\right)$. The Li I $6708 \AA$ line, very weak, is not seen in these models (see Gerbaldi et al. 1995; King et al. 1997 for a detailed analysis of the synthetic spectrum of the Li I line in normal in A, F and solar type stars).

Note: even if such synthetic spectra are only valid for Main Sequence stars, they help us identifying some interesting features in our HAeBe spectra. Indeed, in addition to the Li I $6708 \AA$ line, other absorption lines were found to be indicator of a T Tauri companion. Noticeably, we see in Fig. 2 that the absorption feature at $6678 \AA$ is firstly identified as the Fe I $6677.989 \AA$ line in latetype stars, and then as the He I $6678.154 \AA$ line when the stellar temperature increases towards earlier-type stars. 


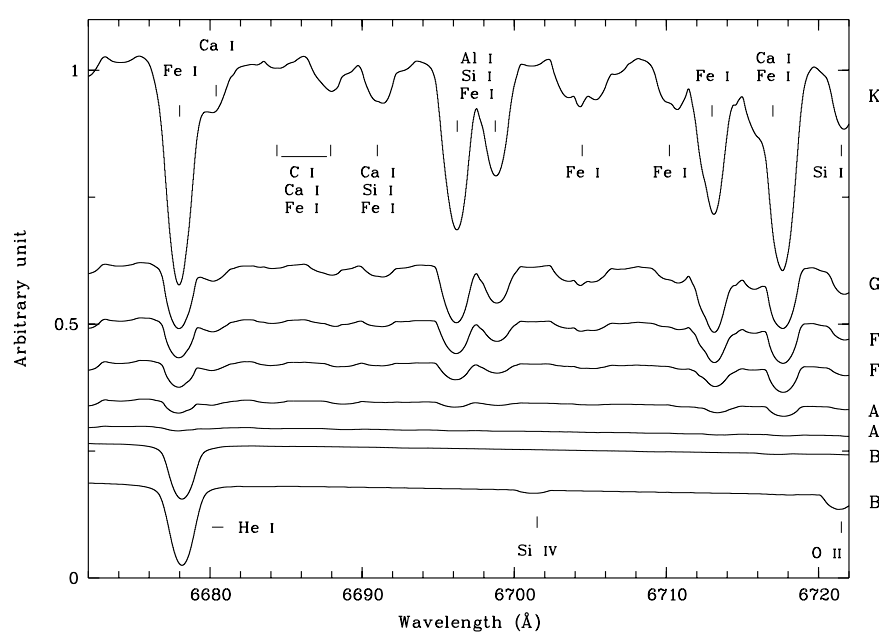

Fig. 2. Kurucz models for solar metallicity stars of various spectral types $\left(V_{\text {rot }}=50 \mathrm{~km} \mathrm{~s}^{-1}\right)$ in the Li I $6708 \AA$ region

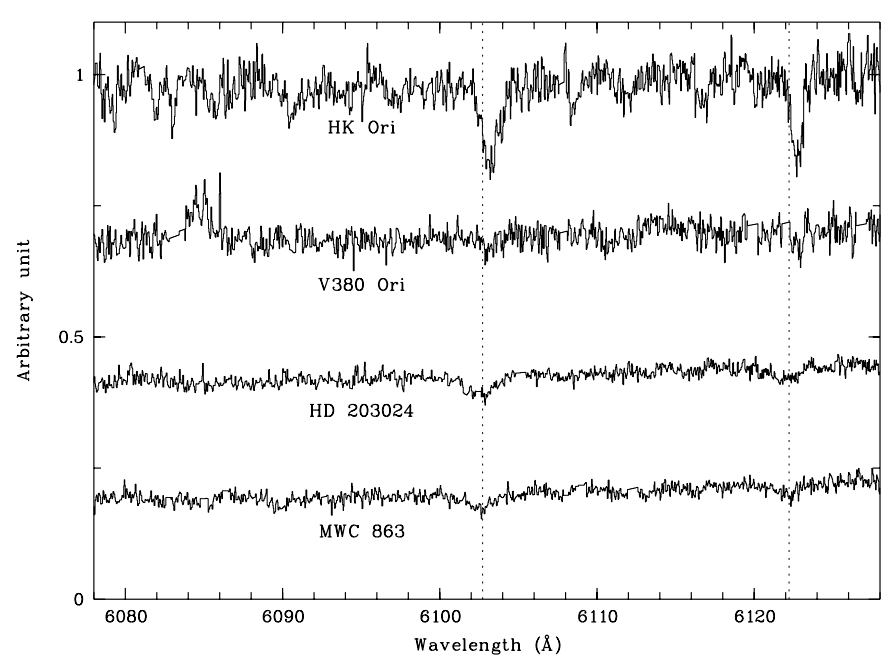

Fig. 3. Ca I lines of HAeBe candidates identified as binaries with positive Li I detection (laboratory positions are shown with doted lines)

The Ca I $6717.681 \AA$ line is seen in late-type stars but not in hotter stars. Thus, the presence in a HAeBe spectrum of the Fe I 6678 and Ca I $6718 \AA$ lines, even if harder to detect that the Li I line, are other features that sign the existence of a cooler companion (see the typical case of HK Ori in Fig. 1).

Figure 3 shows the Li I $6103.65 \AA$ line region for the HAeBe stars with positive Li I $6708 \AA$ line detection. No evidence of Li I $6104 \AA$ absorption is seen, due to a blend with stronger Fe I and CaI lines (see also Dunkin et al. 1997). The metallic lines Ca I 6102.723 and $6122.217 \AA$ are absent in spectra of A/B type stars (as shown is the synthetic spectra in Fig. 4), but their presence in our HAeBe spectra (Fig. 3) are also an evidence for a second lowermass component.

The second group in Table 2 is composed of 7 stars for which strong evidences of radial velocity variations have

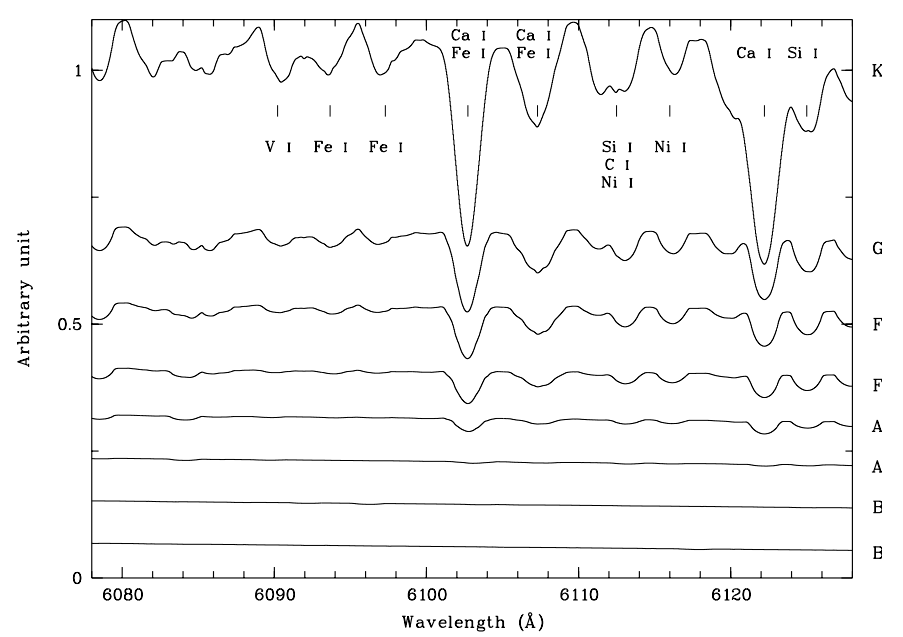

Fig. 4. Kurucz models for solar metallicity stars of various spectral types $\left(V_{\text {rot }}=50 \mathrm{~km} \mathrm{~s}^{-1}\right)$ in the Ca I $6103 \AA$ region

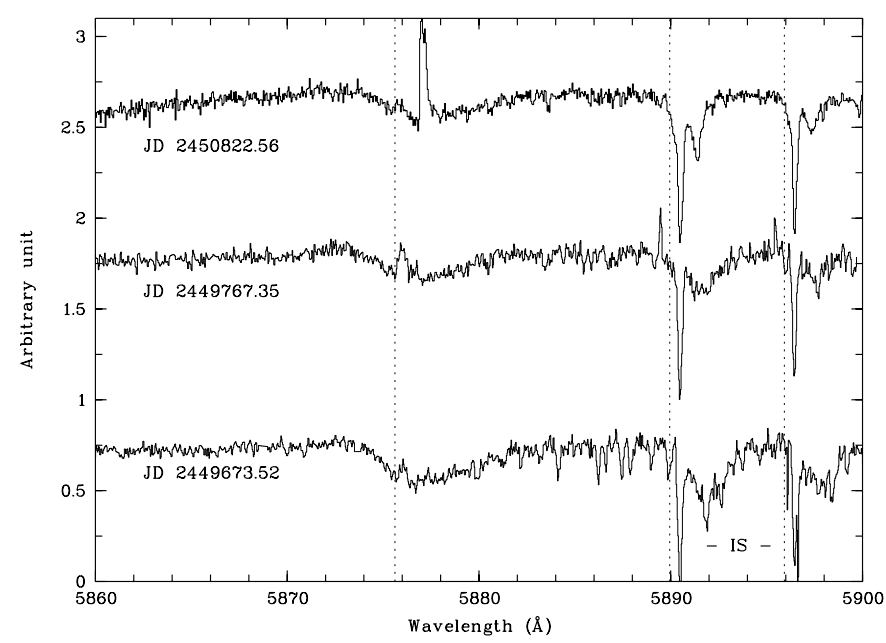

Fig. 5. Spectra of T Ori in the region of He I 5875.621, Na I 5889.951 and $5895.924 \AA$ (doted lines show their laboratory position) at three different JDs (Julian Day). Radial velocity variations are present in the broad photospheric He I lines and Na I lines, as well as in the He I emission feature. Note the strong interstellar Na I absorption lines indicated (IS)

been recorded. If a radial velocity curve and an orbital period could be proposed from our observations, the star is indicated as SB1 for single-lined spectroscopic binary. Figures 5 to 13 show the spectra of stars with radial velocity variations; velocity measurements are gathered in Table 3. For two stars (HD 53367 and AS 442), tentative radial velocity curves and orbital solutions have been obtained using a modified version of the program from Corporon et al. (1996).

For those stars known to be members of visual binary systems, we give in the last column of Table 2 their separation $\rho_{\text {vis }}$. For the visual pairs showing radial velocity variations, this separation $\rho_{\text {vis }}$ is probably not related to the spectroscopic binary separation $\rho_{\text {spec }}<\rho_{\text {vis }}$ : a third component is likely involved in those systems, making them 
hierarchical multiple systems. Each star is discussed in details in Sect. 4.

\subsection{Detected binaries among T2-T5 sample}

Table 4 gives the binary systems spectroscopically detected among $\mathrm{HAeBe}$ candidates from Tables 2 to 5 of the catalogue of The et al. (1994). Note that MWC 623 spectrum in the Li I $6708 \AA$ region, already published in Zickgraf \& Stahl (1989), is not presented here.

Figures 14 to 16 present the spectra for the spectroscopic binaries V361 Ori and HD 199603 and a preliminary velocity curve for the spectroscopic binary V361 Ori.

\subsection{HAeBe candidates undetected as spectroscopic binaries}

Table 5 reports all the negative results for Li I line or radial velocity variations. Some stars with insufficient data to detect orbital motion are marked with "_" in the corresponding column.

Figures 17 and 18 show the Li I 6708 and Ca I $6103 \AA$ regions for some $\mathrm{HAeBe}$ candidates (to be compared with

Table 3. HAeBe candidates detected as spectroscopic binaries, through radial velocity variability. Errors are $5 \mathrm{~km} \mathrm{~s}^{-1}$ for HD 53367, MWC 300, AS 442, MWC 361, and $10 \mathrm{~km} \mathrm{~s}^{-1}$ for T Ori, MWC 1080. A symbol " $*$ indicates a line with emission

\begin{tabular}{|c|c|c|c|}
\hline Star & $\begin{array}{c}\text { JD } \\
(-2400000)\end{array}$ & $\begin{array}{l}\text { observed } \\
\text { line }\end{array}$ & $\begin{array}{r}V_{\text {rad }} \\
\left(\mathrm{km} \mathrm{s}^{-1}\right)\end{array}$ \\
\hline T Ori & 49673.52 & He I 5876 & +101.6 \\
\hline $\mathrm{T}$ Ori & 49673.52 & He I $5876^{\star}$ & +21.2 \\
\hline T Ori & 49673.52 & Na I 5890 & +99.2 \\
\hline T Ori & 49673.52 & Na I 5896 & +95.4 \\
\hline $\mathrm{T}$ Ori & 49675.47 & Не I 5876 & +85.1 \\
\hline T Ori & 49675.47 & He I $5876^{\star}$ & +18.5 \\
\hline T Ori & 49675.47 & Na I 5890 & +79.9 \\
\hline $\mathrm{T}$ Ori & 49767.35 & Не I 5876 & +87.9 \\
\hline T Ori & 49767.35 & He I $5876^{\star}$ & +19.0 \\
\hline T Ori & 49767.35 & Na I 5890 & +83.0 \\
\hline T Ori & 49767.35 & Na I 5896 & +86.3 \\
\hline T Ori & 50822.56 & He I 5876 & +116.4 \\
\hline $\mathrm{T}$ Ori & 50822.56 & He I $5876^{\star}$ & +75.1 \\
\hline T Ori & 50822.56 & Na I 5890 & +71.5 \\
\hline T Ori & 50822.56 & Na I 5896 & +70.7 \\
\hline HD 53367 & 49475.46 & He I 6678 & +25.1 \\
\hline HD 53367 & 49476.51 & Не і 6678 & +26.6 \\
\hline HD 53367 & 49477.49 & He I 5875 & +29.2 \\
\hline HD 53367 & 49647.83 & He I 6678 & +29.9 \\
\hline HD 53367 & 49648.75 & He I 5875 & +34.6 \\
\hline HD 53367 & 49673.60 & He I 4471 & +46.2 \\
\hline HD 53367 & 49673.60 & He I 5875 & +49.1 \\
\hline HD 53367 & 49673.60 & He I 6678 & +47.1 \\
\hline HD 53367 & 49676.72 & He I 4471 & +46.0 \\
\hline HD 53367 & 49676.72 & Не I 5875 & +52.3 \\
\hline
\end{tabular}

Table 3. continued

\begin{tabular}{|c|c|c|c|}
\hline Star & $\begin{array}{c}\text { JD } \\
(-2400000) \\
\end{array}$ & $\begin{array}{l}\text { observed } \\
\text { line }\end{array}$ & $\begin{array}{r}V_{\text {rad }} \\
\left(\mathrm{km} \mathrm{s}^{-1}\right) \\
\end{array}$ \\
\hline HD 53367 & 49769.45 & He I 4471 & +40.0 \\
\hline HD 53367 & 49769.45 & He I 5875 & +45.8 \\
\hline HD 53367 & 49769.45 & He I 6678 & +44.8 \\
\hline HD 53367 & 49821.52 & He I 6678 & +32.3 \\
\hline HD 53367 & 50087.77 & He I 6678 & +59.2 \\
\hline HD 53367 & 50089.69 & Не I 6678 & +56.3 \\
\hline HD 53367 & 50589.46 & He I 6678 & +53.4 \\
\hline HD 53367 & 50821.67 & He I 6678 & +36.0 \\
\hline HD 53367 & 50822.73 & He I 6678 & +35.1 \\
\hline MWC 300 & 49493.52 & He I 5875 & -36.3 \\
\hline MWC 300 & 49493.52 & He I 6678 & -33.9 \\
\hline MWC 300 & 49493.52 & He I $5875^{\star}$ & +46.4 \\
\hline MWC 300 & 49493.52 & He I $6678^{\star}$ & +39.5 \\
\hline MWC 300 & 49591.39 & He I 5875 & -29.2 \\
\hline MWC 300 & 49591.39 & He I 6678 & -32.9 \\
\hline MWC 300 & 49591.39 & He I $5875^{\star}$ & +49.9 \\
\hline MWC 300 & 49591.39 & He I $6678^{\star}$ & +45.3 \\
\hline MWC 300 & 49875.50 & He I 5875 & -43.9 \\
\hline MWC 300 & 49875.50 & He I 6678 & -38.3 \\
\hline MWC 300 & 49875.50 & He I $5875^{\star}$ & +36.6 \\
\hline MWC 300 & 49875.50 & He I $6678^{\star}$ & +27.7 \\
\hline MWC 300 & 49879.47 & He I 5875 & -57.4 \\
\hline MWC 300 & 49879.47 & Не I 6678 & -47.0 \\
\hline MWC 300 & 49879.47 & He I $5875^{\star}$ & +37.0 \\
\hline MWC 300 & 49879.47 & He I $6678^{*}$ & +34.0 \\
\hline MWC 300 & 50293.38 & He I 5875 & -39.4 \\
\hline MWC 300 & 50293.38 & He I 6678 & -29.1 \\
\hline MWC 300 & 50293.38 & He I $5875^{\star}$ & +42.0 \\
\hline MWC 300 & 50293.38 & He I $6678^{\star}$ & +32.6 \\
\hline MWC 300 & 50633.51 & He I 5875 & -16.7 \\
\hline MWC 300 & 50633.51 & He I 6678 & -13.7 \\
\hline MWC 300 & 50633.51 & He I $5875^{\star}$ & +60.5 \\
\hline MWC 300 & 50633.51 & He I $6678^{\star}$ & +55.2 \\
\hline MWC 300 & 50636.42 & He I 5875 & -25.0 \\
\hline MWC 300 & 50636.42 & He I 6678 & -17.0 \\
\hline MWC 300 & 50636.42 & He I $5875^{\star}$ & +61.1 \\
\hline MWC 300 & 50636.42 & He I $6678^{\star}$ & +58.4 \\
\hline AS 442 & 49590.53 & Mg II 4481 & -30.8 \\
\hline AS 442 & 49590.53 & Si II 6347 & -28.8 \\
\hline AS 442 & 49590.53 & Si II 6371 & -33.9 \\
\hline AS 442 & 49674.30 & Mg II 4481 & -13.2 \\
\hline AS 442 & 49674.30 & Si II 6347 & -12.7 \\
\hline AS 442 & 49674.30 & Si II 6371 & -15.6 \\
\hline AS 442 & 49677.36 & Mg II 4481 & -1.5 \\
\hline AS 442 & 49677.36 & Si II 6347 & -5.1 \\
\hline AS 442 & 49677.36 & Si II 6371 & -4.1 \\
\hline AS 442 & 49939.41 & Mg II 4481 & +3.3 \\
\hline AS 442 & 49939.41 & Si II 6347 & +9.3 \\
\hline AS 442 & 49939.41 & Si II 6371 & +6.9 \\
\hline AS 442 & 49952.61 & Mg II 4481 & +2.6 \\
\hline AS 442 & 49952.61 & Si II 6347 & +0.8 \\
\hline AS 442 & 49952.61 & Si II 6371 & +5.0 \\
\hline AS 442 & 50637.52 & Mg II 4481 & -4.2 \\
\hline AS 442 & 50637.52 & Si II 6347 & -8.4 \\
\hline AS 442 & 50637.52 & Si II 6371 & -6.3 \\
\hline AS 442 & 50638.49 & Mg II 4481 & -1.5 \\
\hline AS 442 & 50638.49 & Si II 6347 & -7.8 \\
\hline
\end{tabular}


Table 3. continued

\begin{tabular}{|c|c|c|c|}
\hline Star & $\begin{array}{c}\text { JD } \\
(-2400000)\end{array}$ & $\begin{array}{l}\text { observed } \\
\text { line }\end{array}$ & $\begin{array}{r}V_{\mathrm{rad}} \\
\left(\mathrm{km} \mathrm{s}^{-1}\right)\end{array}$ \\
\hline AS 442 & 50638.49 & Si II 6371 & -7.0 \\
\hline MWC 361 & 49591.62 & He I 4471 & -10.9 \\
\hline MWC 361 & 49591.62 & Mg II 4481 & -21.7 \\
\hline MWC 361 & 49591.62 & Не I 5875 & -3.3 \\
\hline MWC 361 & 49591.62 & He I 6678 & -17.0 \\
\hline MWC 361 & 49595.45 & He I 4471 & -12.2 \\
\hline MWC 361 & 49595.45 & Mg II 4481 & -18.3 \\
\hline MWC 361 & 49595.45 & Не I 5875 & -11.4 \\
\hline MWC 361 & 49595.45 & Не I 6678 & -17.0 \\
\hline MWC 361 & 49597.41 & He I 4471 & -14.0 \\
\hline MWC 361 & 49597.41 & Mg II 4481 & -19.4 \\
\hline MWC 361 & 49675.36 & He I 4471 & -11.3 \\
\hline MWC 361 & 49675.36 & Mg II 4481 & -22.9 \\
\hline MWC 361 & 49675.36 & Не I 5875 & -14.6 \\
\hline MWC 361 & 49675.36 & Не I 6678 & -17.4 \\
\hline MWC 361 & 49875.59 & He I 4471 & -10.7 \\
\hline MWC 361 & 49875.59 & Mg II 4481 & -17.4 \\
\hline MWC 361 & 49875.59 & He I 5875 & -8.0 \\
\hline MWC 361 & 49875.59 & Не I 6678 & -15.7 \\
\hline MWC 361 & 49940.53 & He I 4471 & -8.1 \\
\hline MWC 361 & 49940.53 & Mg II 4481 & -25.4 \\
\hline MWC 361 & 49940.53 & Не I 5875 & -7.9 \\
\hline MWC 361 & 49940.53 & Не I 6678 & -14.9 \\
\hline MWC 361 & 49968.41 & He I 4471 & -7.6 \\
\hline MWC 361 & 49968.41 & Mg II 4481 & -11.0 \\
\hline MWC 361 & 49968.41 & He I 5875 & -8.9 \\
\hline MWC 361 & 49968.41 & He I 6678 & -15.0 \\
\hline MWC 361 & 50017.25 & Не г 6678 & -27.0 \\
\hline MWC 361 & 50293.60 & Не I 4471 & +2.2 \\
\hline MWC 361 & 50293.60 & Mg II 4481 & +2.7 \\
\hline MWC 361 & 50293.60 & He I 5875 & +6.6 \\
\hline MWC 361 & 50635.56 & He I 4471 & -1.1 \\
\hline MWC 361 & 50635.56 & Mg II 4481 & -5.8 \\
\hline MWC 361 & 50635.56 & He I 5875 & -0.3 \\
\hline MWC 361 & 50635.56 & Не г 6678 & -5.2 \\
\hline MWC 361 & 50638.50 & He I 4471 & -0.5 \\
\hline MWC 361 & 50638.50 & Mg II 4481 & -5.4 \\
\hline MWC 361 & 50638.50 & Не I 5875 & +0.9 \\
\hline MWC 361 & 50638.50 & He I 6678 & -3.2 \\
\hline MWC 1080 & 49594.58 & He I 5875 & -297.8 \\
\hline MWC 1080 & 49676.45 & He I 5875 & -207.7 \\
\hline MWC 1080 & 49949.60 & He I 5875 & -190.1 \\
\hline MWC 1080 & 49953.59 & He I 5875 & -285.8 \\
\hline
\end{tabular}

Table 4. Detected spectroscopic binaries among T2-T5 sample

\begin{tabular}{lcccl}
\hline Star & $\begin{array}{c}\text { Li I line } \\
\text { detection }\end{array}$ & $\begin{array}{c}V_{\text {rad }} \\
\text { variations }\end{array}$ & $\begin{array}{c}\text { Thé's } \\
\text { table }\end{array}$ & $\begin{array}{l}v \sin i \\
\left(\mathrm{~km} \mathrm{~s}^{-1}\right)\end{array}$ \\
\hline V361 Ori & $\mathrm{N}$ & $\mathrm{SB1}$ & T5 & 40 \\
MWC 623 & $\mathrm{Y}$ & $\mathrm{N}$ & T4a & \\
HD 199603 & $\mathrm{N}$ & $\mathrm{SB1}$ & $\mathrm{T} 5$ & 60 \\
\hline
\end{tabular}

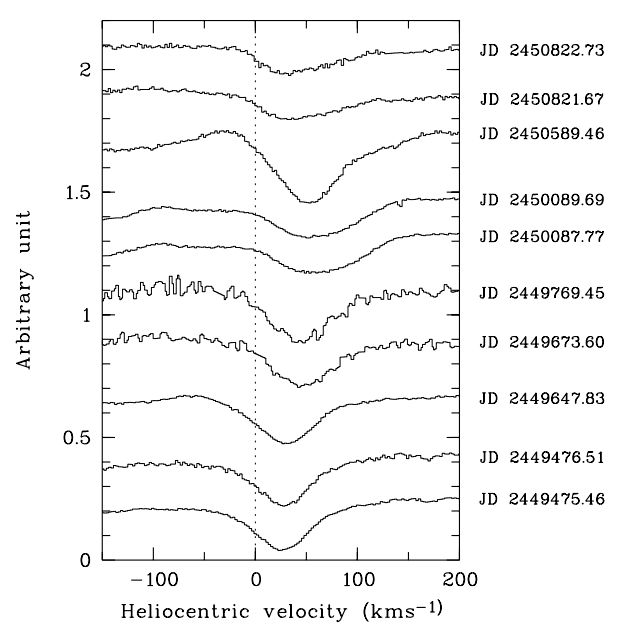

Fig. 6. He I $6678 \AA$ line of HD 53367 at various JDs with radial velocity variations

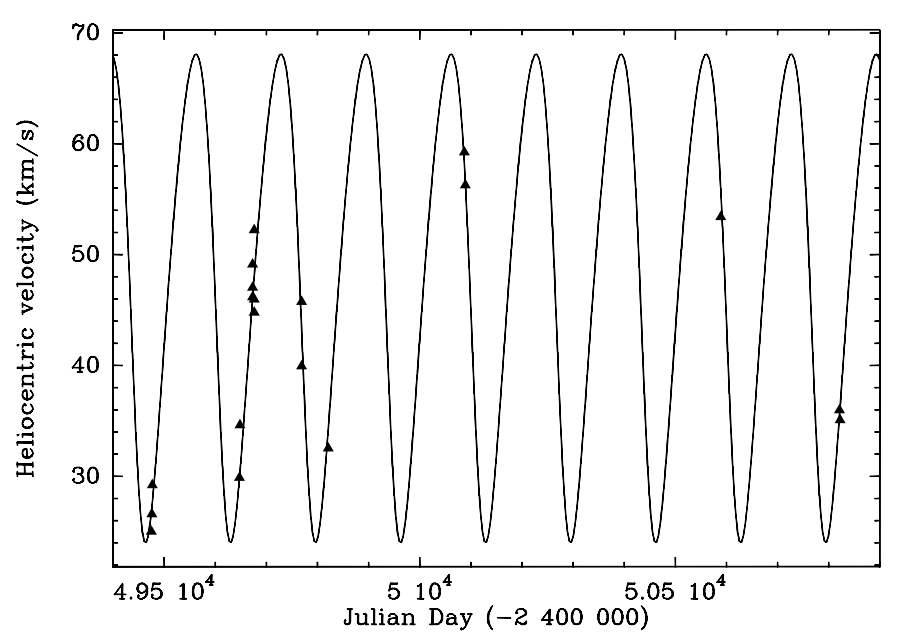

Fig. 7. Heliocentric radial velocity versus Julian Day for HD 53367 showing the temporal spread of our observations. Error bars on the individual points are $5 \mathrm{~km} \mathrm{~s}^{-1}$

Figs. 1 and 3 which show binary stars). Figure 19 shows the He I $6678 \AA$ line of GU CMa with strong variability.

\subsection{Undetected spectroscopic binaries among T2-T5 sample}

Table 6 reports all the negative results for $\mathrm{Li}$ I search or radial velocity variations searches for the T2-T5 sample.

\subsection{Some stars misclassified as $H A$ eBe candidates or doubtful cases}

Table 7 presents some stars that, according to the presently available spectra, are very unlikely to be HAeBe candidates. 


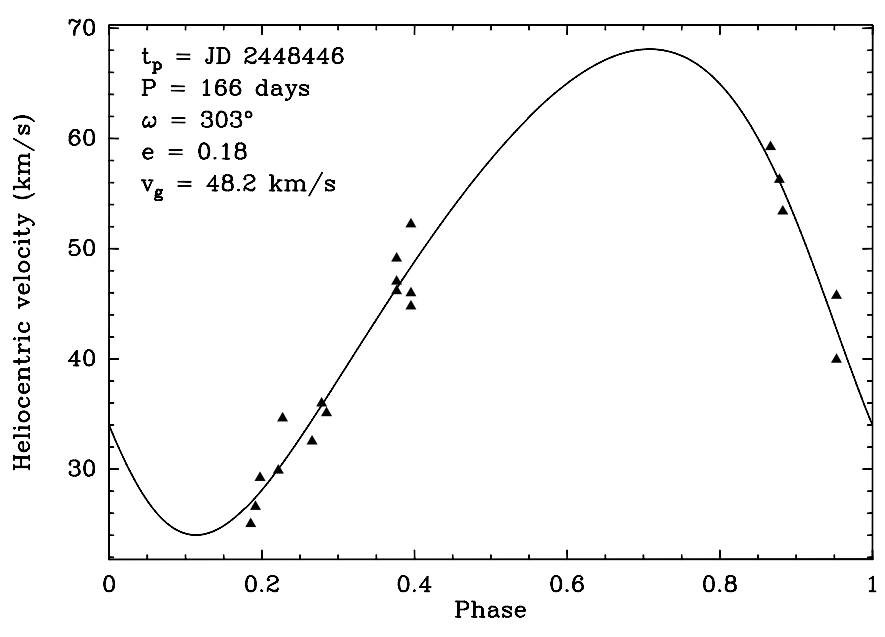

Fig. 8. Velocity curve for HD 53367. Error bars on the individual points are $5 \mathrm{~km} \mathrm{~s}^{-1}$, parameters for the proposed solution are overplotted

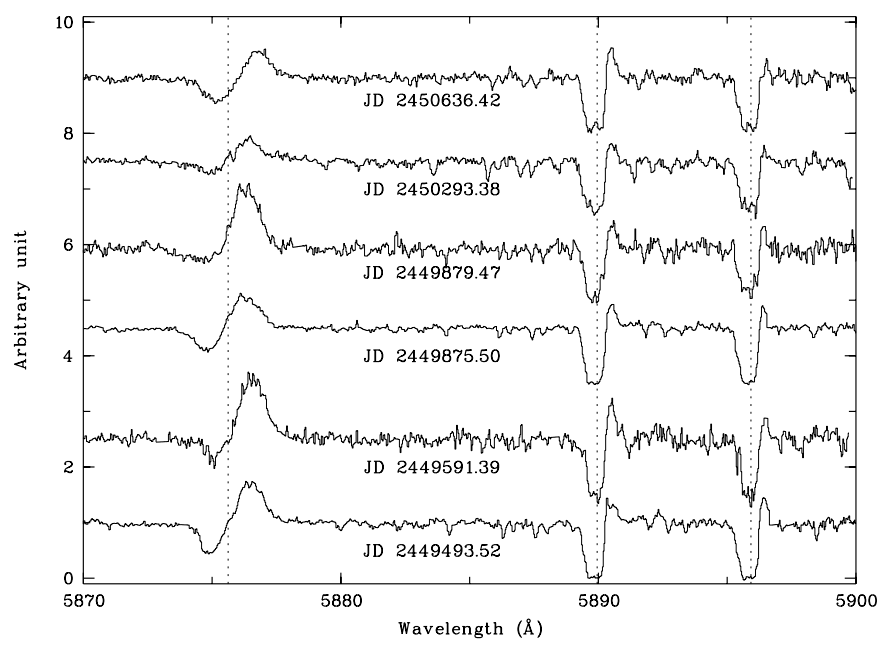

Fig. 9. Spectra of MWC 300 in the region of He I 5875.621 , $\mathrm{Na}$ I 5889.951 and $5895.924 \AA$ (doted lines show their laboratory position) at six different JDs. Radial velocity variations are present in the broad photospheric He I and Na I lines

\section{Notes on individual sources}

HK Ori: a cooler companion is detected thanks to the strong Li I $6708 \AA$ line and other metallic lines. This was previously found by Davis et al. (1981). The rotational velocity of the companion is $\approx 20 \pm 3 \mathrm{~km} \mathrm{~s}^{-1}$, the equivalent width (EW) of the Li line is $170 \pm 10 \mathrm{~mA}$.

The broad He I $6678 \AA$ line from the primary is blended with the Fe I $6680 \AA$ absorption line from the secondary. No radial velocity variations were recorded during our spectroscopic survey.

HK Ori is also known to be a visual binary (separation $\rho_{\text {vis }}=0.34^{\prime \prime}$, see Leinert et al. 1997b). An important point is that the $\mathrm{Li}$ I detection and the presence of Ca I 6103 and $6122 \AA$ lines of the companion confirms its

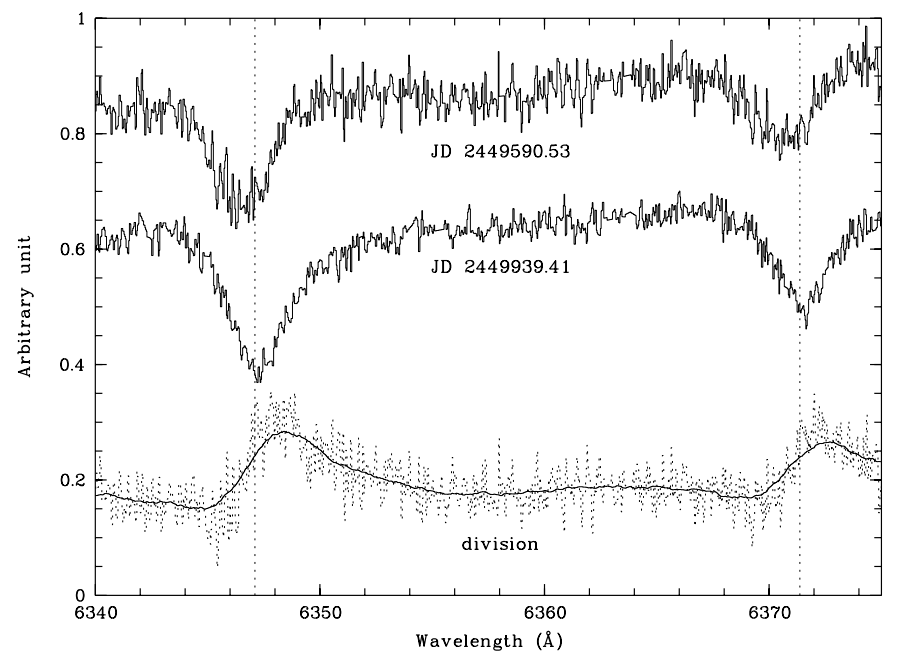

Fig. 10. Si II 6347.109 and $6371.37 \AA$ doublet lines in AS 442 at two different JDs. The division of both spectra figures the radial velocity variation of the doublet lines due to orbital motion

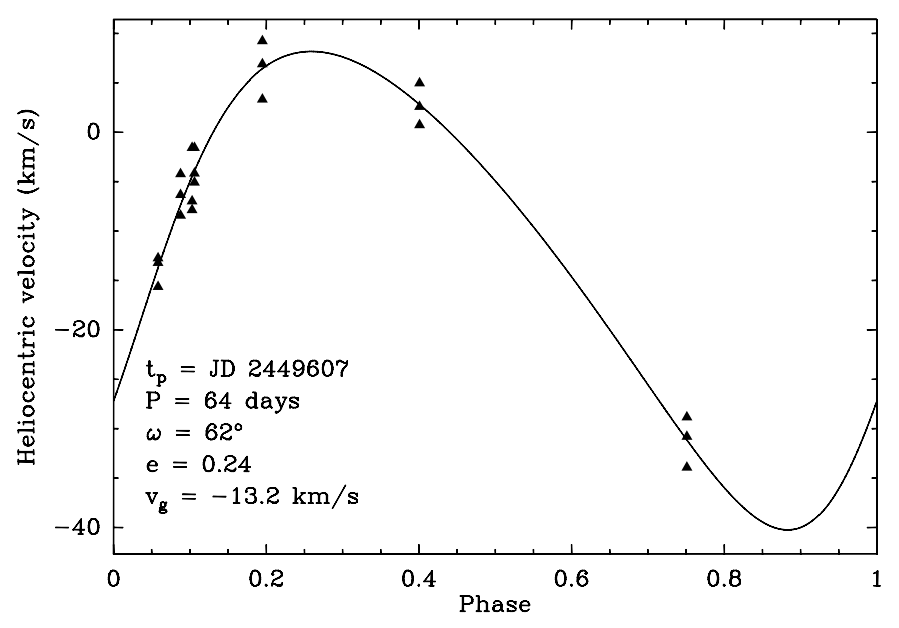

Fig. 11. Same as Fig. 8 for AS 442

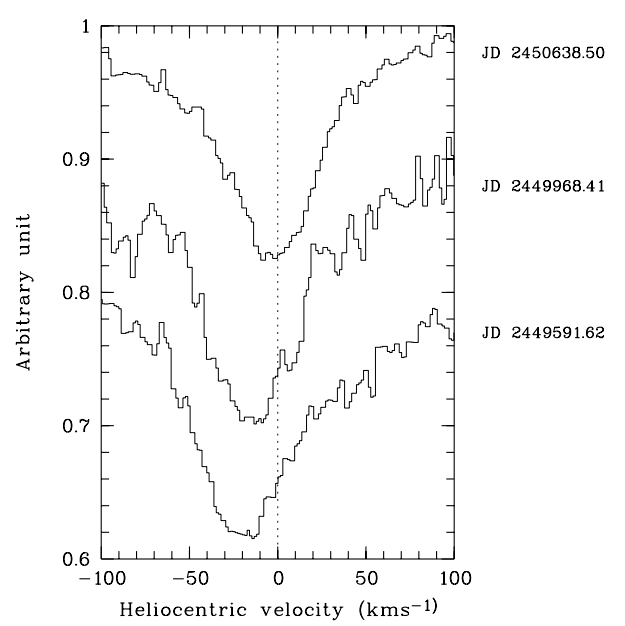

Fig. 12. Radial velocity variations of the MWC 361 He I $6678 \AA$ line 


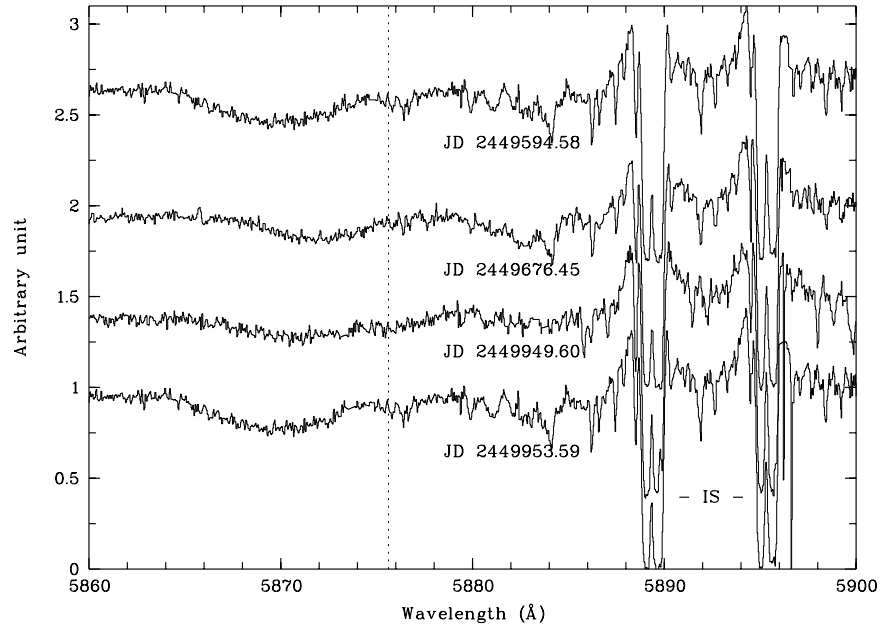

Fig. 13. Spectra of MWC 1080 in the region of He I $5875.621 \AA$ (doted lines show its laboratory position) at four different JDs. Radial velocity variations are present in the very broad photospheric He I lines. Strong interstellar Na I absorption lines are indicated (IS)

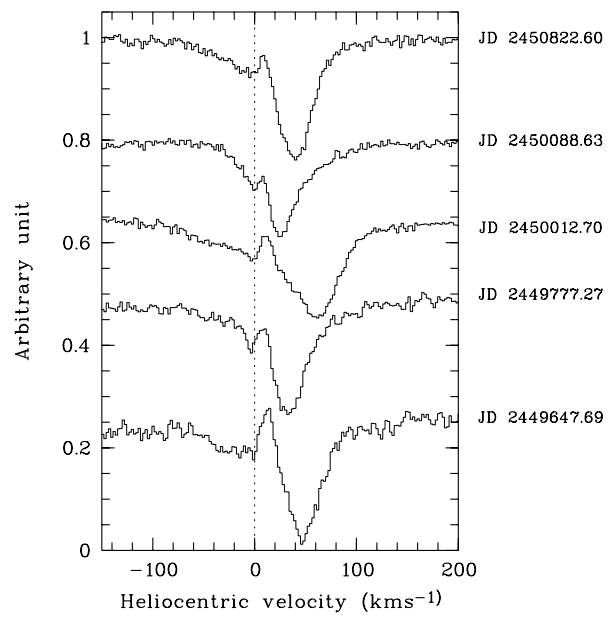

Fig. 14. Same as Fig. 12 for V361 Ori

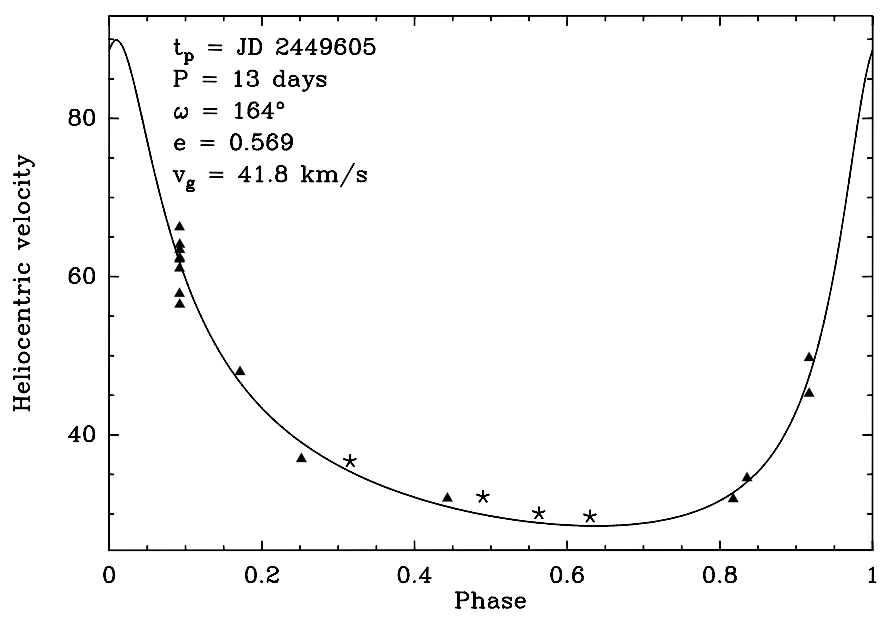

Fig. 15. Same as Fig. 8 for V361 Ori. Star symbols represent overplotted data from Abt et al. (1991)

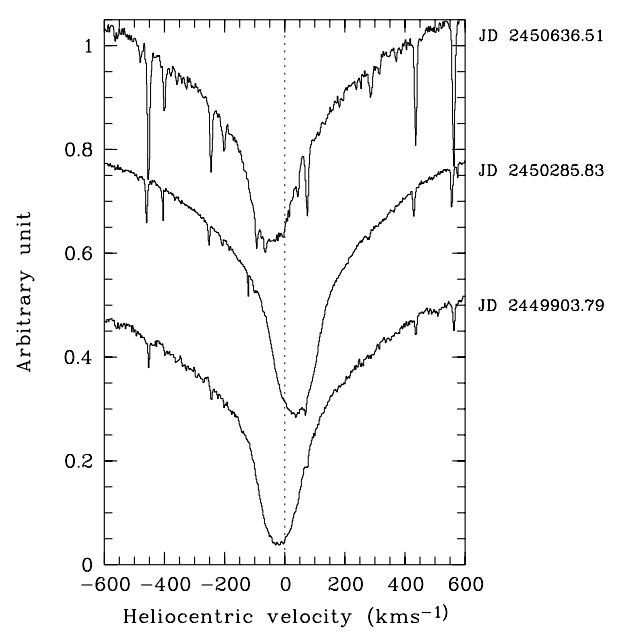

Fig. 16. H $\alpha 6563 \AA$ line of HD 199603 at various JDs. Variations in radial velocity are seen. Narrow absorption lines are due to atmospheric $\mathrm{H}_{2} \mathrm{O}$

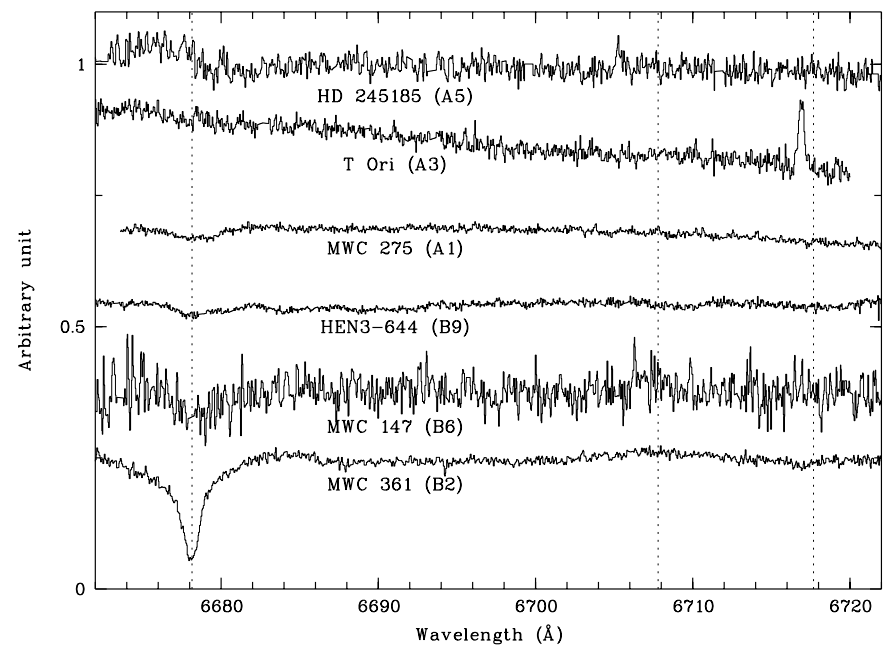

Fig. 17. HAeBe without Li I $6708 \AA$ line detection

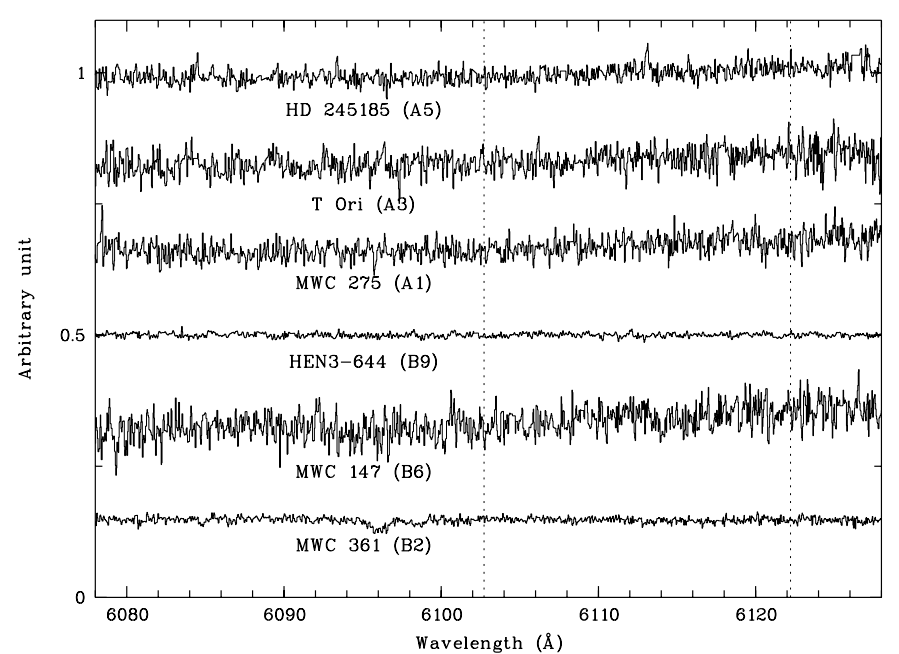

Fig. 18. HAeBe (without Li I $6708 \AA$ line) around Ca I 6103 and $6122 \AA$ 
Table 5. HAeBe candidates undetected as spectroscopic binary. ( $v \sin i$ references in Table 2)

\begin{tabular}{lccl}
\hline Star & $\begin{array}{c}\text { Li l line } \\
\text { detection }\end{array}$ & $\begin{array}{c}V_{\text {rad }} \\
\text { variations }\end{array}$ & $\begin{array}{l}v \sin i \\
\left(\mathrm{~km} \mathrm{~s}^{-1}\right)\end{array}$ \\
\hline HD 245185 & $\mathrm{N}$ & $\mathrm{N}$ & 150 \\
BF Ori & $\mathrm{N}$ & - & $100^{\mathrm{e}}$ \\
HD 250550 & $\mathrm{N}$ & $\mathrm{N}$ & $110^{\mathrm{e}}$ \\
LKHA 215 & $\mathrm{N}$ & - & $60^{\mathrm{e}}$ \\
MWC 147 & $\mathrm{N}$ & $\mathrm{N}$ & $90^{\mathrm{e}}$ \\
GU CMa & $\mathrm{N}$ & $\mathrm{N} ?$ & 150 \\
HEN 3-225 & $\mathrm{N}$ & $\mathrm{N}$ & $110^{\mathrm{f}}$ \\
HD 97048 & $\mathrm{N}$ & $\mathrm{N}$ & $140^{\mathrm{e}}$ \\
HEN 3-331 & $\mathrm{N}$ & $\mathrm{N}$ & 50 \\
HEN 3-554 & $\mathrm{N}$ & - & \\
HEN 3-644 & $\mathrm{N}$ & - & \\
HEN 3-672 & $\mathrm{N}$ & - & \\
HEN 3-692 & $\mathrm{N}$ & - & \\
HD 141569 & $\mathrm{N}$ & $\mathrm{N}$ & 200 \\
HEN 3-1141 & $\mathrm{N}$ & $\mathrm{N}$ & 50 \\
HR 5999 & $\mathrm{N}$ & - & $180^{\mathrm{e}}$ \\
MWC 275 & $\mathrm{N}$ & - & $120^{\mathrm{f}}$ \\
VV Ser & $\mathrm{N}$ & - & \\
MWC 614 & $\mathrm{N}$ & $\mathrm{N}$ & 60 \\
WW Vul & $\mathrm{N}$ & $\mathrm{N}$ & 125 \\
HD 190073 & $\mathrm{N}$ & $\mathrm{N}$ & 20 \\
BD+40 4124 & $\mathrm{N}$ & $\mathrm{N}$ & $180^{\mathrm{f}}$ \\
V361 Cep & $\mathrm{N}$ & $\mathrm{N}$ & $180^{\mathrm{e}}$ \\
BD+46 3471 & $\mathrm{N}$ & $\mathrm{N}$ & $150^{\mathrm{e}}$ \\
IL Cep & $\mathrm{N}$ & $\mathrm{N}$ & $190^{\mathrm{e}}$ \\
BH Cep & $\mathrm{N}$ & $\mathrm{N}$ & 70 \\
SV Cep & $\mathrm{N}$ & $\mathrm{N}$ & 150 \\
BHJ 71 & $\mathrm{N}$ & $\mathrm{N}$ & 25 \\
\hline
\end{tabular}

Table 6. Undetected spectroscopic binaries in the $\mathrm{T} 2-\mathrm{T} 5$ sample

\begin{tabular}{lccll}
\hline Star & $\begin{array}{c}\text { Li I line } \\
\text { detection }\end{array}$ & $\begin{array}{c}V_{\text {rad }} \\
\text { variations }\end{array}$ & $\begin{array}{l}\text { Thé's } \\
\text { table }\end{array}$ & $\begin{array}{l}v \sin i \\
\left(\mathrm{~km} \mathrm{~s}^{-1}\right)\end{array}$ \\
\hline HD 45677 & $\mathrm{N}$ & - & T3 & $80^{\mathrm{e}}$ \\
HD 50138 & $\mathrm{N}$ & - & T3 & 50 \\
HD 97300 & $\mathrm{N}$ & - & T5 & \\
CD-39 8581 & $\mathrm{N}$ & $\mathrm{N}$ & T4b & 200 \\
HD 158352 & $\mathrm{N}$ & $\mathrm{N}$ & T5 & 100 \\
MWC 925 & $\mathrm{N}$ & - & T4b & \\
MWC 930 & $\mathrm{N}$ & $\mathrm{N}$ & T4b & 100 \\
MWC 953 & $\mathrm{N}$ & $\mathrm{N}$ & T4b & 30 \\
AS 321 & $\mathrm{N}$ & $\mathrm{N}$ & T4b & \\
MWC 314 & $\mathrm{N}$ & $\mathrm{N}$ & T4b & \\
MWC 342 & $\mathrm{N}$ & $\mathrm{N}$ & T4a & \\
MWC 1021 & $\mathrm{N}$ & $\mathrm{N}$ & T4b & 70 \\
MWC 1044 & $\mathrm{N}$ & $\mathrm{N}$ & T4b & 200 \\
MWC 655 & $\mathrm{N}$ & $\mathrm{N}$ & T4b & 200 \\
MWC 657 & $\mathrm{N}$ & $\mathrm{N}$ & T4b & 100 \\
MWC 1072 & $\mathrm{~N}$ & $\mathrm{~N}$ & T4b & 200 \\
\hline
\end{tabular}

${ }^{\text {a }}$ This object (= HER 4636) is a visual binary (Chelli et al. 1995) with separation $\rho_{\text {vis }}=4^{\prime \prime}$; individual components were observed spectroscopically and remarks apply for each star.

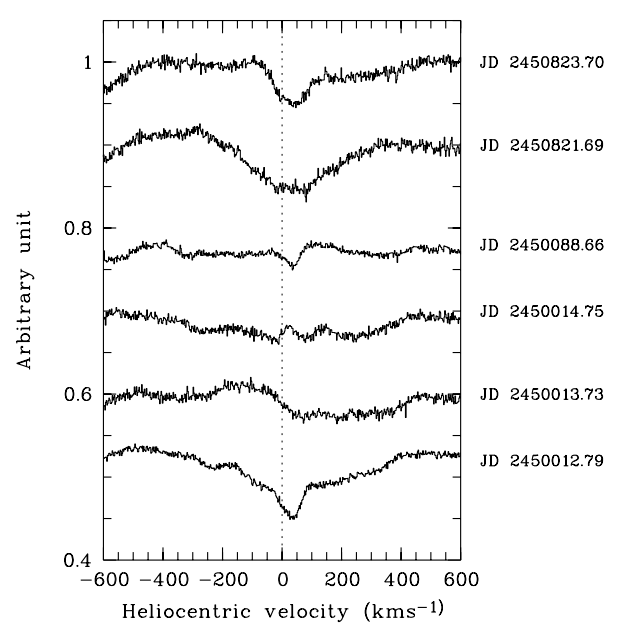

Fig. 19. He I $6678 \AA$ line of GU CMa at various JDs. Variations in radial velocity and intensity are observed

Table 7. Stars rejected as HAeBe or doubtful case

\begin{tabular}{lcccl}
\hline Star & $\begin{array}{c}\text { Li l line } \\
\text { detection }\end{array}$ & $\begin{array}{c}V_{\text {rad }} \\
\text { variations }\end{array}$ & $\begin{array}{c}\text { Thé's } \\
\text { table }\end{array}$ & $\begin{array}{l}v \sin i \\
\left(\mathrm{~km} \mathrm{~s}^{-1}\right)\end{array}$ \\
\hline RY Ori & $\mathrm{Y}$ & $\mathrm{N}$ & $\mathrm{T} 2$ & 50 \\
T Cha & $\mathrm{Y}$ & $\mathrm{Y} ?$ & T2 & 50 \\
HD 199603 & $\mathrm{N}$ & $\mathrm{Y}$ & T5 & 60 \\
MWC 314 & $\mathrm{N}$ & $\mathrm{N}$ & T4b & \\
\hline
\end{tabular}

youth and lower mass. In the discussion of the system by Leinert et al. (1997b), the case a) seems appropriate: the companion is a T Tauri star. A more detailed study of this interesting HAeBe binary system will be given in Bouvier et al. (1998).

V380 Ori: as already claimed by Corcoran \& Ray (1995), Li I line is present in its spectrum: the companion has a rotational velocity of $30 \pm 5 \mathrm{~km} \mathrm{~s}^{-1}, \mathrm{EW}=80 \pm$ $10 \mathrm{~m} \AA$ for the Li I line. Ca I 6103 and Ca I $6122 \AA$ absorption lines from the companion are also detected. V380 Ori is a visual binary $\left(\rho_{\text {vis }}=0.15^{\prime \prime}\right)$ as well. An analysis of its characteristics will be presented in Bouvier et al. (1998).

V586 Ori: while the He I $6678 \AA$ line, due to the HAeBe primary, is clearly visible and broad, we also detect for the first time weak $\mathrm{Li}$ I and Ca I $6717 \AA$ lines, indicative of a cooler companion. Bouvier et al. (1998) indeed confirm the presence of a $\mathrm{T}$ Tauri companion at a separation of $\rho_{\text {vis }}=1^{\prime \prime}$. Secondary rotational velocity is about $30 \mathrm{~km} \mathrm{~s}^{-1}$ and Li I EW is around $50 \mathrm{~m} \AA$.

NX Pup (A+B): this star is in fact a triple system, consisting of a close binary $\left(\rho_{\text {vis }}=0.13^{\prime \prime}\right.$, components $\mathrm{A}+\mathrm{B}$, Bernacca et al. 1993; Brandner et al. 1995) associated to a distant companion $\left(\rho_{\mathrm{vis}}=7^{\prime \prime}\right)$ (component $\mathrm{C}$ ). We spectroscopically observe the close binary for the fist time.

While they detected Li I line in companion C, Brandner et al. (1995) failed to detect lithium in the $\mathrm{A}+\mathrm{B}$ pair. Our spectrum clearly shows for the first time this line in the binary system, confirming the youth of the system as 
proposed by Schoeller et al. (1996) on the basis of high angular resolution optical and near infrared images. Both Li I $(F W H M=1.5 \AA, E W=120 \mathrm{m \AA})$ and Ca I lines are rather broad ( $F W H M=2 \AA)$, and may results from a blend of line from both components.

Higher S/N spectra with high resolution are needed to further investigate the spectral type of both components.

HD 203024: the Li I line has an EW of $70 \mathrm{~mA}$, the rotational velocity of the low-mass companion (first spectroscopic detection) is $\approx 40 \mathrm{~km} \mathrm{~s}^{-1}$. HD 203024 is also a visual binary with separation $\rho_{\text {vis }}=0.3^{\prime \prime}$ (Bouvier et al. 1998).

MWC 863: a cool companion is revealed in $\mathrm{Li} \mathrm{I}$ as well as in other CaI line (6717, 6103 and $6122 \AA)$. The $\mathrm{Li}$ I has an $\mathrm{EW} \approx 40 \mathrm{m \AA}$, the rotational velocity of the secondary is around $30 \mathrm{kms}^{-1}$. Besides this new spectroscopic detection, MWC 863 is known to be a visual binary with $\rho_{\text {vis }}=1.1^{\prime \prime}$ (Reipurth \& Zinnecker 1993).

TY CrA: this young triple system has been extensively spectroscopically surveyed (Lagrange et al. 1993; Corporon et al. 1994, 1996; Beust et al. 1997) and a recent photometric analysis has been made by Casey et al. (1998) and Vaz et al. (1998). It consists of a close central binary $(P \approx 2.9$ days $)$, with a HAeBe primary star and a lower-mass companion; a third much farther away lowmass component orbits the binary. Both lower mass components show Li I absorption line (see Casey et al. 1995; Corporon et al. 1996).

T Ori: this star has already been reported to be a spectroscopic and eclipsing binary by Shevchenko \& Vitrichenko (1994). With the present available data, we observe the radial velocity variations but are unable to confirm the proposed period $P \approx 14$ days for the binary system. Further study of this interesting object is needed to obtain precise masses and radii as it has been done for TY CrA.

HD 53367: Herbst \& Assousa (1977) and Finkenzeller \& Mundt (1984) already reported radial velocity variations for this star. Here, periodic radial velocity variations are reported for the first time in $\mathrm{He}$ I 4471, 5876, and $6678 \AA$ lines: we propose a period of $P \approx 166$ days and an eccentricity $e \approx 0.18$ for the orbital motion of the binary system. More data are needed to confirm this tentative orbital solution.

MWC 300: emission in the red part of He I 5876 and $6678 \AA$ makes it difficult to measure of the photospheric central absorption, but radial velocity variations are correlated with the Na I 5890 and $5996 \AA$ absorption doublet: a monitoring of this star is needed to provide an estimate of the period, our data being to largely spread in time. This is a first detection.

AS 442: periodic radial velocity variations are found in Mg II $4481 \AA$ doublet as well as in the Si II 6347 and $6371 \AA$ absorption doublet. We found a possible orbital solution with $P \approx 64$ days and $e \approx 0.2$, to be confirmed by other observations. This is a first detection.

MWC 361: some evidences of radial velocity variations are found in various lines such as He I 4471, 5876 , and 6678 and $\mathrm{Mg}$ II $4481 \AA$ but the data at hand are not enough to set any period.

MWC 1080: a photometric period of $P \approx 2.9$ days has been determined by Shevchenko et al. (1994) and may compatible with our spectroscopic observations (see spectra at JD $=2449949.60$ and $\mathrm{JD}=2449953.59$ at nearly opposite phase). Line profile variations complicate our measurements. Note the very high blue-shift of the photospheric lines: Shevchenko et al. (1994) gave a $\gamma$ velocity of $-180 \mathrm{~km} \mathrm{~s}^{-1}$ for the MWC 1080 binary system. Together with $\mathrm{T}$ Ori, it is probably the third eclipsing spectroscopic binary with TY CrA and thus deserves further careful observations to constraint the physical parameters of the system.

None of these seven latest stars but TY CrA show a Li I absorption: the companion must be a low mass star with low luminosity - typically few percents of the primary luminosity. A young massif (HAeBe) companion (thus without Li I) would have on the other hand a higher luminosity and its lines should have been detected, unless highly obscured by dust.

Note that HD 53367, MWC 1080 and MWC 361 have all a visual companion. However, these companions are unlikely to be responsible for the radial velocity variations here observed because of the high separation, but rather a tertiary component may be involved in each visual binary system. If the periodic radial velocity variations of these stars are confirmed, then HD 53367, MWC 1080 and MWC 361 are likely hierarchical multiple systems, as TY CrA.

V361 Ori: emission in the blue part of the He I $6678 \AA$ line is sometimes present, as seen in Fig. 14: this may compromise a good measurement of the central position of the line. However, we also made measurements on He I 4471 and 5875 , and the more "symmetric" Mg II $4481 \AA$ absorption lines. Abt et al. (1991) observed this star (= Brun 760) but considered it to be constant in radial velocity. The velocity curve showed in Fig. 15 is computed with our own measurements; some data (those with the lower sigma) from Abt et al. (1991) are overplotted.

New measurements are however needed to confirm the spectroscopic binarity status of V361 Ori.

MWC 623: Zickgraf \& Stahl (1989) found this star to be a binary system with a Li-rich K star, but this property, confirmed here, was not underlined in Thé et al. (1994).

HD 199603: this star is not a classical HAeBe star, no emission lines are seen in its spectrum. It is a well-known spectroscopic and eclipsing binary of $\beta$ Lyr type with a period of $P=1.58$ days (Pedoussault et al. 1984), a property which was not mentioned in Thé et al. (1994). 
MWC 147: Vieira \& Cunha (1994) classified that this star as a spectroscopic binary with a period $P=1$ year and a circular orbit. They based their study on analysis of $\mathrm{H} \alpha$ spectra, with strong emission: our numerous spectra (15 spectra covering 3 years of observations), do not show any radial velocity variation within the error equal to $5 \mathrm{~km} \mathrm{~s}^{-1}$, neither in $\mathrm{H} \alpha$ nor in other photospheric absorption lines (He I $4471,5876, \mathrm{Mg}$ II $4481 \AA$ ). Its spectroscopic binarity status is then doubtful. Note that the IR companion $\left(\rho_{\mathrm{vis}}=3.1^{\prime \prime}=900 \mathrm{AU}\right.$ at $290 \mathrm{pc}$, distance form HipPARCos data) cannot be responsible for the doubtful radial velocity variations observed by Vieira \& Cunha (1994), as its orbital velocity would be near $2710^{3} \mathrm{~km} \mathrm{~s}^{-1}$ if its orbit is circular.

GU CMa: Figure 19 shows the He I $6678 \AA$ line of GU CMa with strongly variable broad features which could sign the possible transit of a rapidly rotating companion. Other lines He I 4 471, and $5876 \AA$ also show intensity variability, as the $\mathrm{H} \alpha$ line. Adding that Shevchenko et al. (1992) classified its photometric light curve as quasiperiodic, this star should be monitored to study possible circumstellar material transit or chromospheric activity.

IL Cep: Shevchenko \& Vitrichenko (1994) proposed a photometric period of $P=51$ days but found a constant radial velocity within the error equal to $6 \mathrm{~km} \mathrm{~s}^{-1}$, as we do. The eclipsing binary status of this star remains to be confirmed.

RY Ori: the spectral type is F6 as given by SIMBAD: it may be a composite spectrum binary as pointed out by Herbig \& Bell (1988) but we lack good quality spectra in the blue to support this view. The Fig. 21 shows its Li I absorption line: if not due to a companion star, RY Ori is more probably a $\mathrm{T}$ Tauri star.

T Cha: this young PMS star has a G8 spectral type: the Li I line detection confirms its youth (Covino et al. 1997). Possible radial variations may be present, however other data are needed to definitively assert this.

HD 94509: this star shows a composite spectrum in the He I $6678 \AA$ region. Superimposed on the broad He I $6678 \AA$ line, narrow absorption features are seen: we tentatively identified them as Fe I 6677.989 , Ca I 6680.628 and Ca I $6717.681 \AA$ lines from a cooler companion, but the velocity of each element were not coherent: FeI was at $-45 \mathrm{~km} \mathrm{~s}^{-1}$, CaI 6681 line at $-35 \mathrm{~km} \mathrm{~s}^{-1}$ and CaI 6717 line at $-7 \mathrm{~km} \mathrm{~s}^{-1}$. Furthermore Li I is not detected, making doubtful the identification with a young cool companion. However, remembering that HD 94509 is an A0I shell star (Reed \& Beatty 1995), we may consider that the narrow absorption features are metallic lines formed in the shell. If the strong absorption is due to the He I $6678 \AA$ line from the shell, its velocity is around $-50 \mathrm{~km} \mathrm{~s}^{-1}$, while if the two others narrow absorption features are from Fe I 6677.989 and $6715.383 \AA$ their respective velocity are $+90 \pm 10 \mathrm{~km} \mathrm{~s}^{-1}$. Small radial velocity variations may also be present from one spectrum to another. This star need further study to precise its evolutionary status.

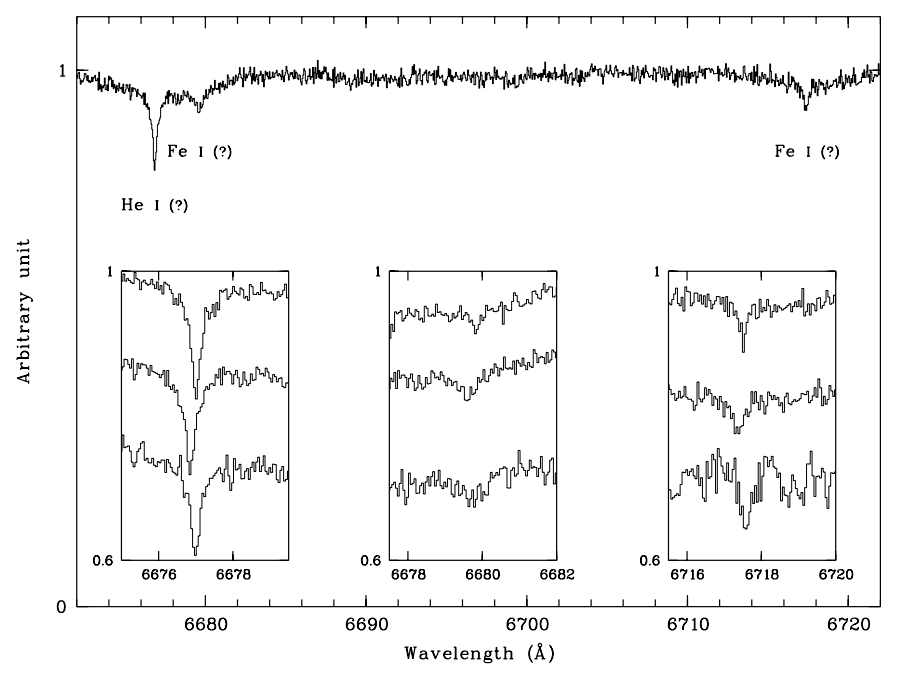

Fig. 20. Spectrum of HD 94509, showing a broad HeI $6678.154 \AA$ line and other narrow metallic lines, probably originating from the shell. The zoomed windows display the three different narrow lines at JD $=2450821.76,2450588.48$ and 2449822.53 (from top to bottom). Variations in radial velocity are observed

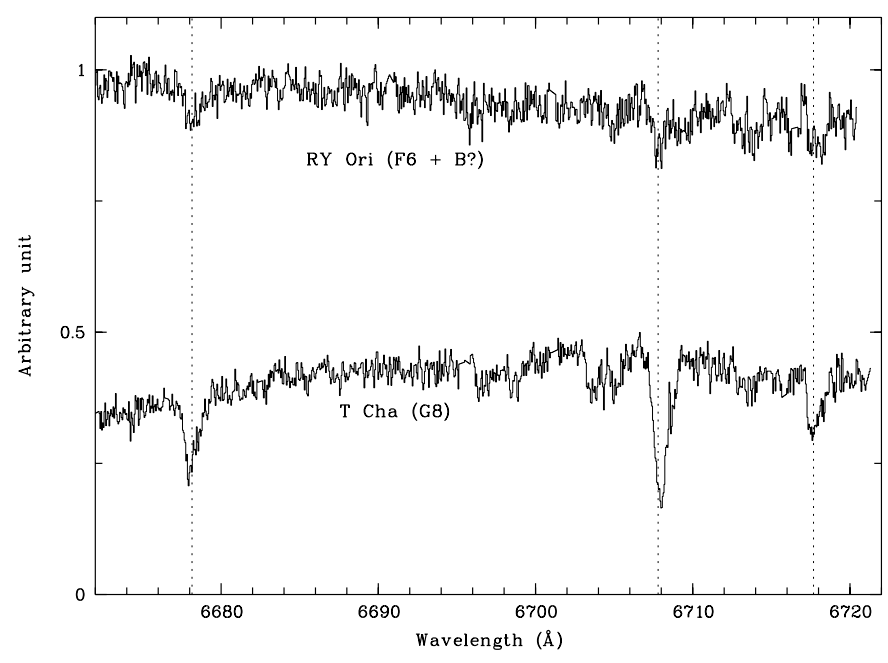

Fig. 21. Doubtful HAeBe (with Li I $6708 \AA$ line detection)

MWC 314: Miroshnichenko (1996) made a detailed analysis of this star and proposed it to be a LBV candidate: MWC 314 is very unlikely a HAeBe star. Our data do not suggest neither the presence of radial velocity variations.

\section{Binary frequency among HAeBe stars}

In the course of this survey, we detected 6 binary systems through Li absorptions and 7 spectroscopic binaries. Before deriving any physical information, we need to address the important question of biases. Several biases have been identified and are therefore discussed below. 


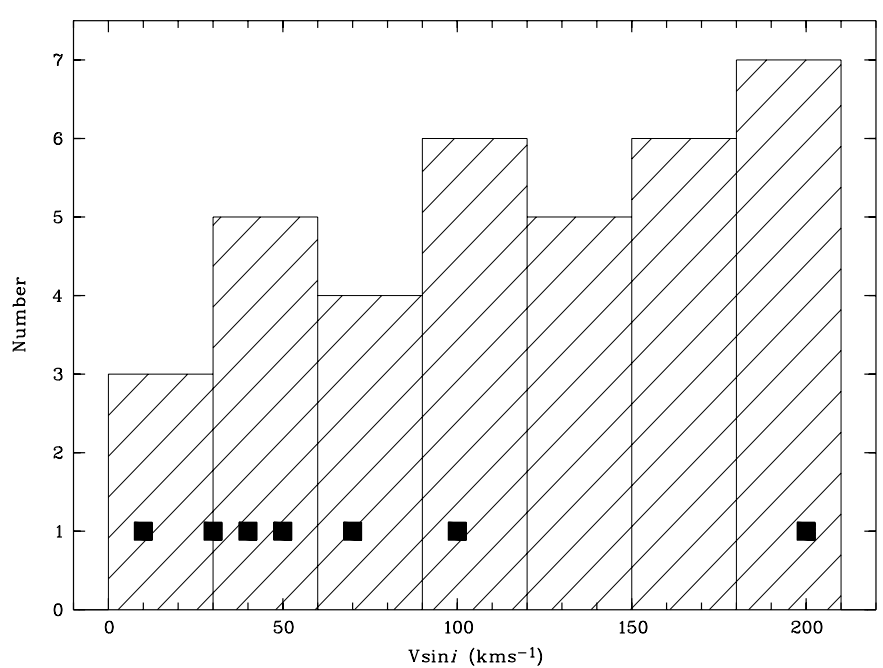

Fig. 22. The distribution of $v \sin i$ for the observed HAeBe from Table 1 of The et al. (1994). The bin size is $30 \mathrm{~km} \mathrm{~s}^{-1}$, filled squared represent spectroscopic binaries with radial velocity variations from Table 2

\subsection{Possible biases}

\subsubsection{Rotational velocity}

Herbig Ae/Be stars show usually a broad distribution in $v \sin i$, up to $300 \mathrm{~km} \mathrm{~s}^{-1}$ (Grady et al. 1996). The higher rotational velocity of the HAeBe primary the more difficult it is to detect radial velocity variations. The projected rotational velocities of our stars are displayed in last column of Tables 2 and 5 for HAeBe in T1 sample, and in Tables 4 and 6 for T2-T5 sample. $v \sin i$ measurements were obtained by visually comparing some photospheric lines (mainly He I 4471 and $6678 \AA$ ) with synthetic spectra broadened by rotation (Kurucz 1993; Hubeny et al. 1994). Error on such estimation should not exceed $\pm 30 \mathrm{~km} \mathrm{~s}^{-1}$. For some stars, information was lacking (not enough spectral lines or imprecise spectral type). Our results were finally compared with previously published measurements (Davis et al. 1983; Finkenzeller 1985; Böhm \& Catala 1995; Grady et al. 1996), when available. They appeared to be always consistent with them.

Restricting ourself to HAeBe from T1 sample, Fig. 22 shows the histogram of the $v \sin i$. The projected rotational velocity for the stars ranges from $10 \mathrm{~km} \mathrm{~s}^{-1}$ (TY CrA) to $200 \mathrm{~km} \mathrm{~s}^{-1}$ (V380 Ori, HD 141569, MWC 1080). Interestingly enough, we see that all stars with observed radial velocity variations (symbolized with a filled square) have a $v \sin i$ below $100 \mathrm{~km} \mathrm{~s}^{-1}$, but MWC 1080 . So, among the 16 stars with $v \sin i<100 \mathrm{~km} \mathrm{~s}^{-1}, 6$ are spectroscopic binaries, i.e. a binary frequency of $37.5 \%$. If we apply this ratio to the 20 remaining high-rotators $(100<$ $v \sin i<200 \mathrm{~km} \mathrm{~s}^{-1}$ ), we should expect to detect 6 to 7 more spectroscopic binaries, in addition to MWC 1080. Note that we have not included in this scheme the 5 (ap-

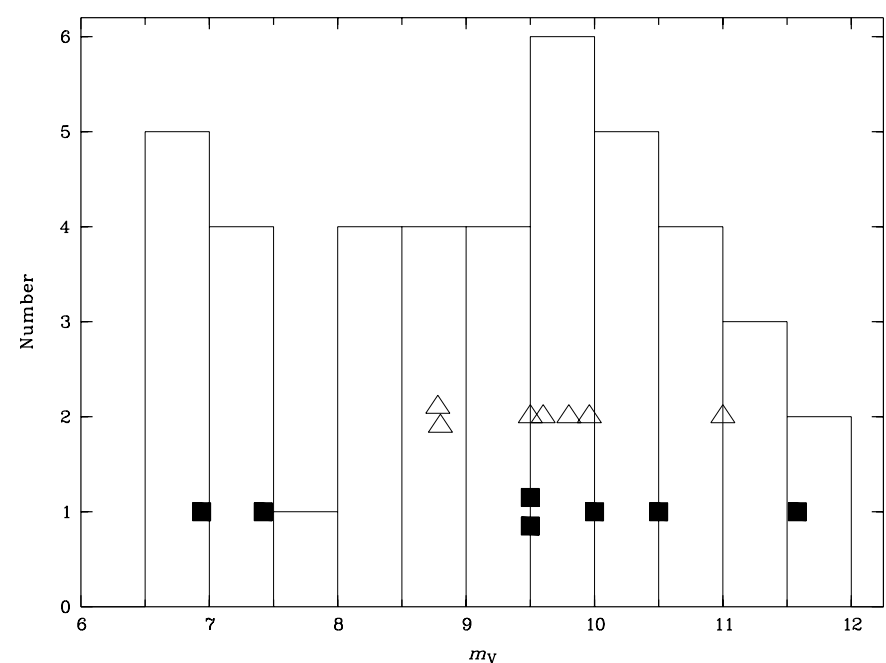

Fig. 23. The distribution of $V$ magnitude for the observed HAeBe from Table 1 of Thé et al. (1994). Filled squared represent spectroscopic binaries with radial velocity variations from Table 2, while open triangles represent spectrum binaries with Li I line detection. Note that TY CrA $(V=9.5)$ shows both radial velocity variations and Li I absorption from companions

parently) non-binary stars with unknown $v \sin i$ (probably larger than $100 \mathrm{~km} \mathrm{~s}^{-1}$ ).

Finally, if the $\mathrm{T}$ Tauri companion is a fast rotator $\left(V_{\text {rot }}>50-100 \mathrm{~km} \mathrm{~s}^{-1}\right)$, its Li I line could be more difficult to detect. This argument may however be compensated by the fact that usually rapid rotators show a larger abundance of Li I than slow rotators do (see Soderblom et al. 1993; Martin et al. 1994; Cunha et al. 1995; Jones et al. 1996) at least for young G and early-K stars; on the other hand, Duncan \& Rebull (1996) found no strong correlation between Li I and $v \sin i$ for young stars in Orion.

If the system is composed of two HAeBe stars with similar luminosities, the blend of the lines (broadened by high rotational velocities) will make difficult their radial velocity measurement.

In conclusion, we estimate that we may have missed at least $50 \%$ of the spectroscopic binary with radial velocity variations.

\subsubsection{Luminosity ratio}

If the primary HAeBe star is much more luminous than its $\mathrm{T}$ Tauri companion $\left(\Delta m_{V}<4-5\right)$, the Li I line (among other lines) from the secondary companion is obviously very difficult to detect. So the binary criterion will be the radial velocity variations of the primary, if any.

Figure 23 shows the $m_{V}$ histogram for stars in T1 sample. HAeBe binary systems with radial velocity variations (filled squares) are rather well distributed between $m_{V}=6.5$ and $m_{V}=11$. For spectrum binaries (with Li I line absorption from a cooler component), there may be a lack of detection if $m_{V}<8.5$. Keeping the same binary 
frequency as for fainter stars ( 7 spectrum binaries including TY CrA among 28 stars with $m_{V}>8.5$ ), up to 4 binaries may have been missed among the 14 remaining brightest stars.

For spectrum binary systems composed of two HAeBe stars, Li I criterion is not anymore valid to probe the duplicity. Bouvier and collaborators (Bouvier et al. 1998; Corporon 1998) have detected at least 5 pairs of gravitationally linked HAeBe among the 30 visual binary systems (separation $\rho>0.13^{\prime \prime}$ ) observed with Adaptive Optics. Assuming a similar ratio for much smaller binary separations, we could expect to detect 1 or 2 new spectroscopic binaries composed of two HAeBe stars (this estimate is however a lower limit as not all spectral type for visual companions in Bouvier et al. study could have been determined).

\subsubsection{Branch effect:}

This effect has already been described and we showed that it was not important for binaries with one high mass component and one low mass companion. The 6 systems detected through $\mathrm{Li}$ absorptions belong to this category. For the 7 other spectroscopic binary systems, the secondary spectral type is unknown in most cases. However, the total luminosity of those systems is much lower than our limiting magnitude $\left(m_{V}<11\right)$, except for the faintest star MWC 1080: we may have included this spectroscopic binary in our survey because the secondary contributes to a non-negligible part of the system luminosity. This issue deserves further studies.

Considering our whole sample, we claim finally that the Branch effect won't affect our preliminary binary frequency estimate for HAeBe stars.

\subsubsection{Conclusion on biases:}

In conclusion, at least $50 \%$ of the spectroscopic binaries could have been missed because of either rotational difficulties measurements or the luminosity ratio.

\subsection{Derived binary frequency}

Table 2 contains 13 Herbig Ae/Be spectroscopic binaries. If we only consider the Doppler shift of the lines criterion, we have 7 spectroscopic binary systems (6 stars from the second group of our Table 2 plus the TY CrA system) among the $42 \mathrm{HAeBe}$ stars of our principal sample: so our observed binary frequency $f b$ is $\approx 17 \%$. This is a lower limit: for reasons stated above, the true spectroscopic binary frequency for HAeBe may be as high as $35 \%$.

Restricting ourself to secure or candidate spectroscopic binary systems with $P<100$ days (namely T Ori, AS 442, MWC 1080 and TY CrA), the binary frequency is $10 \%$. For short-period ( $P<100$ days) WTTS spectroscopic binaries, Mathieu (1992) found a binary frequency $f b=11 \pm 4 \%$, slightly higher than for MS solar-mass stars $f b=7 \pm 2 \%$ found by Duquennoy \& Mayor (1991). Our present binary frequency estimate for short period systems seems comparable to the one for T Tauri or MS stars. However, as our number are small and the biases important, this binary frequency for short-period systems should be regarded as a lower limit: future observations (e.g. using interferometric technics) will certainly help to detect new close systems.

\subsection{Is $X$-ray emission a binary criterion?}

We address now the puzzling issue of X-ray emission in HAeBe stars. We may wonder if this property could also be used to identify double stars and thus give an other way of determining the binary frequency.

Herbig $\mathrm{Ae} / \mathrm{Be}$ are known to be strong X-ray emitters (Zinnecker \& Preibisch 1994; Damiani et al. 1994). However, the existence of X-ray emission intrinsical to Herbig Ae/Be stars is still doubtful: these stars indeed lack convective zones that could create a solar-type dynamo and heat a corona via strong magnetic field.

A non-solar dynamo model has been proposed by Tout \& Pringle (1995) and applied to the HAeBe star HD 104237 by Skinner \& Yamauchi (1996): if this shear dynamo model may generate an active corona, the X-ray luminosity $L_{\mathrm{X}}$ predicted seems to be lower than observed. However, many parameters in this model remain free and are not known empirically.

Another possibility is that the X-ray emission detected arises from a cooler $\mathrm{T}$ Tauri companion associated to the HAeBe star (Zinnecker \& Preibisch 1994; Damiani et al. 1994), possibly through a process of colliding winds (Zhekov et al. 1995). In our limited sample of HAeBe binaries (Table 2), 4 stars are known to be X-ray emitters (V380 Ori, TY CrA, MWC 361 and MWC 1080); 2 other binaries (T Ori and HD 53367) have not been detected by EINSTEIN nor ROSAT, while the 7 remaining stars have no known X-ray properties. Thus the apparent trend is that X-ray emission is a possible indicator of binarity for HAeBe stars: this conclusion has also been found in the case of visual HAeBe binaries (Bouvier et al. 1998; Corporon 1998). Nevertheless, it would be worth to observe the 7 remaining binary stars in the X-ray domain.

\section{Conclusion}

In course of our high resolution spectroscopic survey of Herbig $\mathrm{Ae} / \mathrm{Be}(\mathrm{HAeBe})$ binary stars, we observed 42 objects with $m_{V}<11: 6$ stars exhibit Li I $6708 \AA$ absorption line attributed to a cooler companion (4 are new detections), and for 7 other stars, radial velocity variations are recorded (4 are new detections). TY CrA is a 
particular triple system for which both binarity criteria (i.e. radial velocity variation and presence of Li I absorption line) are observed. Four stars unlikely appear to belong to the HAeBe class.

The Li I line, not observed in our HAeBe candidates from Table 5, is not only an indicator of binarity, but may also help in some cases to classify a star as either a T Tauri or as a HAeBe star. Depending on the presence or not of the Li I $6708 \AA$ line, the distinction, between the class of young low mass stars and the class of young intermediate mass stars, could be inferred thanks to this criterion. This distinction has been proposed here for some peculiar cases, such as RY Ori or T Cha.

Within our reduced sample, the observed binary frequency for short-period spectroscopic HAeBe systems $(f b \approx 10 \%)$ is roughly comparable to the one of $\mathrm{T}$ Tauri or MS stars. The true binary frequency is probably higher considering to the present biases working against the detection of spectroscopic binary stars.

Due the limitation in magnitude, this systematic search would be greatly completed with the use of $8 \mathrm{~m}$ class telescope, to have access of fainter HAeBe stars and enlarge our sample, and to interferometric technics under development. It is emphasized that the spectroscopic binaries discussed in the paper deserves further observations in order to obtain more information about the secondary component (luminosity, age) and possibly to retrieve the masses: their careful study by various means (spectroscopic follow-up, careful photometric monitoring, lunar occultation, interferometric measurements...) are encouraged.

Acknowledgements. This research has made use of the Simbad database, operated at CDS, Strasbourg, France. We warmly thanks the INSU and ESO observing programs committees for generous telescope time allocations and the OHP and ESO telescope teams for their kind support. P.C. thanks P. Prugniel for early discussion at OHP and D. Queloz for his efficient and friendly presence during the first nights with ÉLODIE. We would like to express our gratitude towards S. Allain, D. Mouillet, J.C. Augereau and L. Siess who kindly performed some of the observations, and also to C. Ounnas and J. Rodriguez for some service observations. We thank J. Bouvier for providing useful comments, and $\mathrm{H}$. Beust who specially modified his program for this study. Finally, we are grateful to the referee, Dr. Andrea Richichi, for useful comments on this work and careful reading of the manuscript.

\section{References}

Abt H.A., Gomez A.E., Levy S.G., 1990, ApJS 74, 551

Abt H.A., Wang R., Cardona O., 1991, ApJ 367, 155

Baranne A., Queloz D., Mayor M., et al., 1996, A\&AS 119, 390

Bernacca P.L., Lattanzi M.G., Bucciarelli B., et al., 1993, A\&A 278, L47
Beust H., Corporon P., Siess L., Forestini M., Lagrange A.-M., 1997, A\&A 320, 478

Böhm T., Catala C., 1995, A\&A 301, 155

Bouvier J., Corporon P., et al., 1998, A\&A (in preparation)

Branch D., 1976, ApJ 210, 392

Brandner W., Alcala J.M., Kunkel M., Moneti A., Zinnecker H., 1996, A\&A 307, 121

Brandner W., Bouvier J., Grebel E.K., Tessier E., De Winter D., Beuzit J.L., 1995, A\&A 298, 818

Casey B.W., Mathieu R.D., Suntzeff N.B., Lee C.-W., Cardelli J.A., 1993, AJ 105, 2276

Casey B.W., Mathieu R.D., Suntzeff N.B., Walter F.M., 1995, AJ 109, 2156

Casey B.W., Mathieu R.D., Vaz L.P.R., Andersen J., Suntzeff N.B., 1998, AJ 115, 1617

Chelli A., Cruz-Gonzalez I., Reipurth B., 1995, A\&AS 114, 135

Clarke C., 1996, "Evolutionnary processes in binary stars" Wijers A.M.J., Melvyn B.D. and Tout C.A. (ed.), NATO ASI Series, Vol. C 477, p. 31

Corcoran D., Ray T.P., 1995, A\&A 301, 729

Corporon P., 1998, Ph.D. thesis, Université Grenoble I

Corporon P., Lagrange A.-M., Beust H., 1996, A\&A 310, 228

Corporon P., Lagrange A.-M., Bouvier J., 1994, A\&A 282, L21

Covino E., Alcala J.M., Allain S., Bouvier J., Terranegra L., Krautter J., 1997, A\&A 328, 187

Cunha K., Smith V.V., Lambert D.L., 1995, ApJ 452, 634

Damiani F., Micela G., Sciortino S., Harnden F.R.J., 1994, ApJ 436, 807

Davis R., Strom K.M., Strom S.E., 1983, AJ 88, 1644

Davis R.E., Strom S.E., Strom K.M., 1981, BAAS 13, 855

Duncan D.K., Rebull L.M., 1996, PASP 108, 738

Dunkin S.K., Barlow M.J., Ryan S.G., 1997, MNRAS 290, 165

Duquennoy A., Mayor M., 1991, A\&A 248, 524

Figueiredo J., 1997, A\&A 318, 783

Finkenzeller U., 1985, A\&A 151, 340

Finkenzeller U., Mundt R., 1984, ApJS 55, 109

Garmany C.D., Conti P.S., Massey P., 1980, ApJ 242, 1063

Gerbaldi M., Faraggiana R., Castelli F., 1995, A\&AS 111, 1

Ghez A.M., Mc Carthy D.W., Patience J.L., Beck T.L., 1997, ApJ 481, 378

Ghez A.M., Neugebauer G., Matthews K., 1993, AJ 106, 2005

Gillet D., Burnage R., Kohler D., et al., 1994, Ap\&SS 108, 181

Grady C.A., Pérez M.R., Talavera A., et al., 1996, A\&AS 120, 157

Herbig G.H., Bell K.R., 1988, Lick Obs. Bulle. 1111, 1

Herbst W., Assousa G.E., 1977, ApJ 217, 473

Hillenbrand L.A., 1994, in "The nature and evolutionary status of Herbig Ae/Be stars", Thé P.S., Pérez M.R., van den Heuvel E.P.J (eds.), ASP Conf. Ser. 62, 369

Hubeny I., Lanz T., Jeffery C.S., 1994, News1. Anal. Astron. Spectra 20, 30

Jones B.F., Shetrone M., Fischer D., Soderblom D.R., 1996, AJ 112, 186

King J.R., Deliyannis C.P., Hiltgen D.D., Stephens A., Cunha K., Boesgaard A.M., 1997, AJ 113, 1871

Kurucz R.L., 1993, Cambridge, SAO

Lada E.A., Evans N.J., Depoy D.L., Gatley I., 1991, ApJ 371, 171

Lagrange A.-M., Corporon P., Bouvier J., 1993, A\&A 274, 785

Leinert C., Henry T., Glindemann A., Mc Carthy D.W., 1997a, A\&A 325, 159 
Leinert C., Richichi A., Haas M., 1997b, A\&A 318, 472

Leinert C., Zinnecker H., Weitzel N., Christou J., Ridgway S.T., Jameson R., Haas M., Lenzen R., 1993, A\&A 278, 129

Li W., Evans N.J., Harvey P.M., Colome C., 1994, ApJ 433, 199

Martin E.L., 1994, in "The nature and evolutionary status of Herbig Ae/Be stars", Thé P.S., Pérez M.R. et van den Heuvel E.P.J. (eds.), Vol. 62, p. 315

Martin E.L., Rebolo R., Magazzu A., Pavlenko Y.V., 1994, A\&A 282, 503

Mathieu R.D., 1992, in "A Complementary Approaches to Double and Multiple Star Research", McAlister H.A., Hartkopf W.I. (eds.), IAU Colloquium 135, ASP Conf. Ser. 32,30

Mayor M., Duquennoy A., Halbwachs J.L., Mermilliod J.-C., 1992, in "A Complementary Approaches to Double and Multiple Star Research", McAlister H.A., Hartkopf W.I. (eds.), IAU Colloquium 135, ASP Conf. Ser. 32, 73

Miller G.E., Scalo J.M., 1978, PASP 90, 506

Miroshnichenko A.S., 1996, A\&A 312, 941

Morse J.A., Mathieu R.D., Levine S.E., 1991, AJ 101, 1495

Nordström B., Stefanik R.P., Latham D.W., Andersen J., 1997, A\&AS 126, 21

Padgett D.L., Stapelfeldt K.R., 1994, AJ 107, 720

Padgett D.L., Strom S.E., Ghez A., 1997, ApJ 477, 705

Pedoussaut A., Ginestet N., Carquillat J.M., 1984, A\&AS 58, 601

Pirzkal N., Spillar E.J., Dyck H.M., 1997, ApJ 481, 392

Prosser C.F., Stauffer J.R., Hartmann L., Soderblom D.R., Jones B.F., Werner M.W., Mc Caughrean M.J., 1994, ApJ
421,517

Reed B.C., Beatty A.E., 1995, ApJS 97, 189

Reipurth B., Zinnecker H., 1993, A\&A 278, 81

Schoeller M., Brandner W., Lehmann T., Weigelt G., Zinnecker H., 1996, A\&A 315, 445

Shevchenko V.S.N., Grankin K.A., Ibragimov M.B., Kondratiev V.Yu., Melnikov S., 1994, in "The nature and evolutionary status of Herbig Ae/Be stars" Thé P.S., Pérez M.R. et van den Heuvel E.P.J. (eds.), Vol. 62, p. 43

Shevchenko V.S., Grankin N., Ibragimov K.A., Yu. M., Melnikov S., Yakubov D., 1992, Astrophys. Space Sci. 202, 121

Shevchenko V.S., Vitrichenko E.A., 1994, in "The nature and evolutionary status of Herbig Ae/Be stars" Thé P.S., Pérez M.R., van den Heuvel E.P.J. (eds.), Vol. 62, p. 55

Skinner S.L., Yamauchi S., 1996, ApJ 471, 987

Simon M., Ghez A.M., Leinert C., et al., 1995, ApJ 443, 625

Soderblom D.R., Jones B.F., Balachandran S., Stauffer J.R., Duncan D.K., Fedele S.B., Hudon J.D., 1993, AJ 106, 1059

Thé P.S., de Winter D., Perez M.R., 1994, A\&AS 104, 315

Tout C.A., Pringle J.E., 1995, MNRAS 272, 528

Vaz L.P.R., Andersen J., Casey B.W., Clausen J.V., Mathieu R.D., Heyer I., 1998, A\&AS 130, 245

Vieira S.L.A., Cunha N.C.S., 1994, IAU Informational Bulletin of Variable Stars 4090, 1

Walter F.M., Brown A., Mathieu R.D., Myers P.C., Vrba F.J., 1988, AJ 96, 297

Welty A.D., 1995, AJ 110, 776

Zhekov S.A., Palla F., Prusti T., 1995, MNRAS 276, L51

Zickgraf F.J., Stahl O., 1989, A\&A 223, 165

Zinnecker H., Preibisch T., 1994, A\&A 292, 152 